\title{
CAA Slat Noise Studies Applying Stochastic Sound Sources Based On Solenoidal Digital Filters
}

\author{
R. Ewert* \\ DLR, Institute of Aerodynamics and Flow Technology \\ Technical Acoustics, Lilienthalplatz 7, 38108 Braunschweig, Germany \\ e-mail: roland.ewert@dlr.de \\ R. Emunds ${ }^{\dagger}$ \\ Airbus, Aerodynamic Design 83 Data (EGAG) \\ Huenefeldstr. 1-5, 28183, Bremen, Germany
}

\begin{abstract}
The present paper studies the application of a low-cost CAA approach to a slat noise problem. A new fast and cheap stochastic approach is introduced to model the unsteady turbulent sound sources in the slat-cove. It is based on the spatial filtering of a random white-noise field and incorporates information about the integral length scale and the turbulent kinetic energy from a steady RANS computation. The stochastic method, which stems from the LES inflow boundary condition proposed by Klein et al. ${ }^{6}$ that reproduces first- and second-order one-point statistics, is extended in this paper for aeroacoustic applications. The extended formulation yields a perfectly solenoidal velocity field that is capable to reproduce exactly the complete second-order two-point correlation tensor of homogeneous isotropic turbulence. Results for the sound generation at the slat are given for the underlying RANS mean-flow field being based on a Menter SST turbulence model with and without Kato-Launder modification, respectively. The results for the modeled turbulent flow-field and the radiated acoustic field exhibit physical meaningful characteristics.
\end{abstract}

\section{Introduction}

A IRCRAFT noise reduction as achieved through the development and application of high bypass low noise turbofan engines has shifted the focus of interest to airframe noise as an equally important noise source during the approach phase. Experimental studies have identified deployed slats as prominent noise contributors. $^{1,2}$ To reduce noise levels further, numerical tools will become necessary in the future to achieve an optimized low noise design of airframe components. Therefore, the development of cheap and quick computational methods, which can be used in an optimization process, is essential. In the last years numerical techniques evolved in the framework of Computational Aeroacoustics (CAA) that are optimized for the simulation of wave-propagation, i.e., high-order non-dispersive spatial and temporal discretization schemes and high-quality non-reflecting boundary conditions. A key point is how an acoustic solver based on the linearized Euler equations (LEE) or derivatives of it can be used for design-to-noise approaches to airframe component design. A presumably accurate noise prediction methodology arises if appropriate acoustic sources of the

${ }^{*}$ Research Engineer, DLR, Institute of Aeordynamics and Flow Technology, Technical Acoustics, Braunschweig, Germany, AIAA Member.

${ }^{\dagger}$ Engineer, Airbus, Aerodynamic Design \& Data (EGAG), Bremen, Germany

\section{1 of 23}

American Institute of Aeronautics and Astronautics 
propagation equations are computed from time accurate LES or DNS simulations of the turbulent near field. However, such approaches are too expensive to be considered for a design process that demands to evaluate design modifications in short response times.

In this paper the application of a cheaper computational approach to a slat noise problem is studied that involves CAA techniques in conjunction with stochastic sound sources in the time domain ('Low-cost CAA'). A new technique is introduced to generate broadband stochastic sound sources in the time-domain, which is based on the digital filtering of random data. The method is a further development of the LES inflow boundary condition of Klein et al. ${ }^{6}$ that accurately reproduces first- and second-order one-point statistics. The method is extended in this paper to prescribe a fully solenoidal velocity field on a source patch in the interior CAA domain. The solenoidal extension is shown to additionally reproduce exactly the second-order two-point correlation tensor of homogeneous isotropic turbulence in two or three dimensions, respectively. The stochastic method provides some advantage over the classical approach using inverse Fourier transform. It is time- and memory efficient, strictly solenoidal, and can easily be applied to the highly non-uniform mean-flow field in the slat-cove region using local results for the kinetic turbulent energy and the corresponding turbulent length scale from the RANS solution. Furthermore, it avoids the occurrence of shear decorrelations and resolves broadband spectra continously. A further extension to non-homogeneous anisotropic solenoidal flows is possible with the transformation proposed by Smirnov et al. ${ }^{3}$ For the slat noise simulations a modified high-lift configuration with retracted flap is chosen in order to avoid additional sound sources.

Section II gives an overview about the stochastic method used in this paper. Section III describes some details about the CAA method to integrate the acoustic field, and finally, Section IV gives computational results for simulated homogeneous isotropic turbulence and for slat-noise sound generation. The slat-noise computations are based on two turbulence models and are carried out for a Mach number $M=0.10$.

\section{Stochastic sound source modeling}

Starting from a forced linear acoustic wave equation with wave operator $\mathcal{L}$, acoustic variable $p^{\prime}$ and source $Q$ in the time-domain,

$$
\mathcal{L} p^{\prime}(\boldsymbol{x}, t)=Q(\boldsymbol{x}, t)
$$

it is straight forward to express the far-field density of the acoustic variable due to the statistical properties of the source by an integration over the source volume $\boldsymbol{y}^{4}$

$$
\overline{\widetilde{p}^{\prime 2}(\boldsymbol{x}, \omega)}=\int_{\boldsymbol{y}} \int_{\boldsymbol{\xi}} \widetilde{G^{*}}(\boldsymbol{x}, \boldsymbol{y}-\boldsymbol{\xi} / 2, \omega) \widetilde{G}\left((\boldsymbol{x}, \boldsymbol{y}+\boldsymbol{\xi} / 2, \omega) \widetilde{Q}_{12}(\boldsymbol{y}, \boldsymbol{\xi}, \omega) d \boldsymbol{\xi} d \boldsymbol{y}\right.
$$

Here $\widetilde{G}$ and $\widetilde{G^{*}}$ denote the appropriate Green's function and its conjugate complex in the frequency domain, respectively, which are defined by the linear wave-operator of Eq. (1). Furthermore, $\widetilde{Q}_{12}$ is a cross spectral density, which is found from the Fourier transform of the two-point space-time covariance of the source $Q$ of Eq. (1) between source points $\boldsymbol{y}_{1}=\boldsymbol{y}-\boldsymbol{\xi} / 2$ and $\boldsymbol{y}_{2}=\boldsymbol{y}+\boldsymbol{\xi} / 2$ and time separation $\tau$

$$
\begin{gathered}
\widetilde{Q}_{12}(\boldsymbol{y}, \boldsymbol{\xi}, \omega)=\int_{-\infty}^{\infty} R(\boldsymbol{y}, \boldsymbol{\xi}, \tau) e^{i \omega \tau} d \tau, \\
R(\boldsymbol{y}, \boldsymbol{\xi}, \tau)=\overline{Q(\boldsymbol{y}-\boldsymbol{\xi} / 2, t-\tau / 2) Q(\boldsymbol{y}+\boldsymbol{\xi} / 2, t+\tau / 2)} .
\end{gathered}
$$

Eq. (2) is the basis of all statistical noise theories in the frequency domain and is recalled here, because it evidences that the far-field spectrum is completely determined if just the two-point space-time covariance of the sources, Eq. (4), are known. For turbulence related noise sources this means a great reduction of the necessary informations about the causal turbulent flow field, since the turbulent flow field needs an infinite set of m-point nth-order correlations to be uniquely described. ${ }^{5}$ 
Furthermore, a synthetic fluctuating source $\mathrm{Q}(\boldsymbol{x}, \mathrm{t})$ in Eq. (1), which realizes just the proper two-point space-time correlations, Eq. (4), will yield a far-field spectrum, which satisfies Eq. (2). In other words, a synthetic fluctuating source term, that realizes the proper two-point space-time correlations in conjunction with a time-domain method, Eq. (1), gives an alternative way to solve Eq. (2). It will yield an exact solution if the underlying second-order two-point correlations are exactly known.

Since the two-point space-time correlations can be modeled through relatively simple stationary descriptions, ${ }^{4}$ it might be possible to determine them accurately enough for a wide class of flow problems based on a steady state RANS computation. However, the key issue remains, to set up synthetic fluctuating sources that approach in their one- and two-point correlations a predefined target value. The method discussed in the next section is capable to achieve this demand.

\section{A. Stochastic fluctuating fields via digital filtering}

In Ref. ${ }^{6}$ a new method was proposed to generate pseudo turbulent velocities at the inflow plane of a large eddy simulation (LES) via digital filtering of a stochastic white-noise field. The method is able to reproduce prescribed second order one-point statistics. The method is sketched in the following considering a homogeneous one-dimensional problem. In this case the velocity field $u$ follows by spatially weighting a unity white-noise field $\mathcal{U}$ with a filter kernel $G(r)$ :

$$
u(x, t)=\int_{-\infty}^{\infty} G\left(x-x^{\prime}\right) \cdot \mathcal{U}\left(x^{\prime}, t\right) d x^{\prime} .
$$

$G(r)$ is an even function, i.e., $G(r)=G(-r)$. The unity white-noise field has the covariance

$$
\mathcal{R}(x, r)=\overline{\mathcal{U}(x+r, t) \mathcal{U}(x, t)}=\lim _{T \rightarrow \infty} \frac{1}{2 T} \int_{-T}^{T} \mathcal{U}(x+r, t) \mathcal{U}(x, t) d t=\delta(r),
$$

where $\delta$ denotes the Dirac delta function. Inserting Eq. (5) into the definition of the covariance of the velocity fluctuations $R(r):=\overline{u(x+r, t) u(x, t)}$ and using the properties of Eq. (6), the covariance of the filtered velocity field becomes the convolution of the filter kernel with itself

$$
R(r)=\int_{-\infty}^{\infty} G(r-\xi) G(\xi) d \xi=G * G
$$

where $\xi=x^{\prime}-x$ is the relative separation distance between points $x$ and $x^{\prime}$. In general the filter kernel can be weighted with an arbitrary constant. In the following the constant is assumed to be chosen such that the covariance $R(r)$ of Eq. (7) becomes the correlation $R^{0}(r)$ of $u$ with $R^{0}(0)=1$ since

$$
R^{0}(r):=\frac{\overline{u(x+r, t) u(x, t)}}{\overline{u(x, t) u(x, t)}} .
$$

The related filter kernel is labeled $G^{0}(r)$. Due to the convolution, Eq. (7), the spatial Fourier transform of the correlation function follows to be

$$
\widetilde{R}^{0}(k)=\left(\widetilde{G^{0}}(k)\right)^{2},
$$

where $k$ denotes the wave-number, which is related to the spatial coordinate $x$. In other words, if a certain correlation $R^{0}$ has to be achieved, the filter kernel is defined by

$$
\widetilde{G}^{0}(k)=\sqrt{\widetilde{R^{0}}(k)} .
$$

Practically, the filter kernel of any meaningful correlation can be achieved through Eq. (9). However, the filter kernel, which was considered in $^{6}$ and is also used in this paper, is a Gaussian. It is easy to show using 
Eq. (8) that a spatial Gaussian kernel

$$
G^{0}(x)=\exp \left(-\frac{\pi}{2} \frac{x^{2}}{\Delta^{2}}\right)
$$

yields for the correlation again a Gaussian function, but with half the width, i.e.,

$$
R^{0}(x)=\exp \left(-\frac{\pi}{4} \frac{x^{2}}{\Delta^{2}}\right)
$$

The integral length scale of the correlation Eq. (11) follows from this Gaussian correlation to be

$$
L=\int_{0}^{\infty} R^{0}(r) d r=\Delta,
$$

hence the parameter $\Delta$ in Eq. (10) is directly determined by the (local) integral length scale.

On a discrete equidistantly spaced grid of spacing $\Delta x$, the continuous integral of Eq. (5) can be approximated with a finite sum

$$
u_{m}=\sum_{k=-N}^{N} G_{k} r_{k+m},
$$

which has the formal shape of a digital non-recursive filter. In this approximation $u_{m}$ is the velocity at grid point $m$ and $G_{k}$ is the integral mean value of the filter kernel $G(r)$ for an off-center distance $k \Delta x$ and an integration interval $\Delta x$. The filter Kernel $G(r)$ follows from that of a particular correlation function $G^{0}$ by scaling it with an additional factor $\hat{A}$ such that the local value of the velocity covariance is reached. For the Gaussian filter kernel, the discrete filter kernel is approximated by

$$
G_{k} \simeq \hat{A} \exp \left(-\frac{\pi}{2} \frac{k^{2}}{d^{2}}\right)
$$

where $d:=\Delta / \Delta x$ denotes the non-dimensionalized integral length scale. The quantity $r_{m}$ is a random value that follows by integrating the white-noise field over the interval $\Delta x$ :

$$
r_{m}=\int_{-\Delta x / 2}^{\Delta x / 2} \mathcal{U}\left(x^{\prime}\right) d x^{\prime}
$$

and $N$ defines the finite support of the filter. In this work it was found that a cut-off limit $\left|G_{k}^{0}\right| \geq 10^{-2}$, i.e. $N \approx 1.7 d$, yields a sufficient accurate approximation to the integral Eq. (5). The local integration of the white-noise field over $\Delta x$ yields a spectral cut-off of the white-noise spectrum for a wave-length larger than $\Delta x$. The random values exhibit due to Eq. (6) the properties $\overline{r_{m}}=0$ and

$$
\overline{r_{m} r_{k}}=\left\{\begin{array}{lll}
0 & \text { for } & m \neq k \\
\Delta x & \text { for } & m=k
\end{array}\right.
$$

The values for $r_{m}$ are obtained by loading each computational node with an appropriately scaled random number. It follows from Eq. (16) that the amplitude of the random fluctuations $\hat{r}_{m}$ must be proportional to $\sqrt{\Delta x}$. The extension of the filter operation is straight forward. The Gaussian filter has the property to be separable, e.g. in 2-D it can be split according to

$$
G(|\boldsymbol{x}|)=\exp \left(-\frac{\pi}{2} \frac{x^{2}+y^{2}}{\Delta^{2}}\right)=\exp \left(-\frac{\pi}{2} \frac{x^{2}}{\Delta^{2}}\right) \exp \left(-\frac{\pi}{2} \frac{y^{2}}{\Delta^{2}}\right)=G(x) G(y),
$$

such that the multi-dimensional extension is achieved by successively applying the one-dimensional filtering operation in each direction. The procedure is carried out for each velocity component independently. A

$$
4 \text { of } 23
$$


temporal dependence is obtained by introducing an additional temporal dimension and by applying the filtering also for discrete time levels, using an appropriate time scale. The temporal evolution of the random field $r_{m}$ is achieved by shifting the random data of each spatial slice to the next higher time level and by updating the first time level with new random data. Next, the time dependent fluctuating field at the new time level follows by applying the filter sequentially to the updated random field.

\section{B. Stochastic extension for noise simulations: Solenoidal Digital Filtering}

In order to apply the previously sketched method for acoustic noise prediction purposes some extensions have to be considered.

- In an LES approach the stochastically generated data is just used to update the $2 \mathrm{D}$ inflow plane of a 3D problem. To compute acoustic source terms, the method would be applied not only on the boundaries of the computational domain rather than on a certain source patch in the full computational domain. One major concern is, that the genuine method yields a velocity field that is not solenoidal. In the LES approach, due to the additional degree of freedom in the direction normal to the inflow plane, chances are that the incompressible computational problem automatically adapts to a solenoidal solution. In an aeroacoustic application, however, the non-solenoidal velocity field would give rise to spurious acoustic sources.

- The convection of the turbulence related vorticity in non-uniform mean-flow has to be included into the model.

- Finally, the two-point correlations from stochastic filtering also exhibit spherical symmetry due to the spherical symmetric filter kernel. However, it is well known for isotropic turbulence ${ }^{5}$ that the correlation function in lateral direction $g(r)$ differs from that in longitudinal direction $f(r)$, i.e., is not spherical symmetric.

While $f(r)$ might be well described by a Gaussian shape, $g(r)$ has a different length scale and even becomes negative for a certain range of $r$. In order to solve the aforementioned problems the following modifications have been considered:

1. The white-noise field of Eq. (5) is convected frozen along the streamline of the mean-flow field with the local mean-flow velocity.

2. In $2 \mathrm{D}$, a scalar streamfunction $\psi$ is modeled by the digital filtering procedure rather than the velocity field itself.

Hence, the 2D convolution integral for the stochastically modeled fluctuating streamfunction $\psi$ becomes

$$
\psi(\boldsymbol{x}, t)=\int_{\boldsymbol{x}^{\prime}} G\left(\left|\boldsymbol{x}-\boldsymbol{x}^{\prime}\right|, \Delta\left(\boldsymbol{x}^{\prime}\right)\right) \mathcal{U}\left(\boldsymbol{x}^{\prime}\right) d \boldsymbol{x}^{\prime},
$$

where $\boldsymbol{x}^{\prime}$ denotes the source region. The appearance of $\Delta\left(\boldsymbol{x}^{\prime}\right)$ shall indicate the dependence of the filter kernel width on the local length scale in the source region, assuming a sufficiently small variation of the local length scale. A variation of the length scale too strong would lead to a deviation of the local correlation function from the target value. ${ }^{6}$ The reason is that Eq. (8) is based on a constant local filter width. From the scalar streamfunction the velocity field follows via

$$
u_{\psi}^{\prime}=\frac{\partial \psi}{\partial y}, \quad v_{\psi}^{\prime}=-\frac{\partial \psi}{\partial x},
$$

which is strictly solenoidal. The meaning of $\mathcal{U}\left(\boldsymbol{x}^{\prime}\right)$ in Eq. (17) needs some more clarification: Firstly, a length parameter $f$ along the streamline can be introduced, which is up to a constant $c$ proportional to the elapsed

$$
5 \text { of } 23
$$

American Institute of Aeronautics and Astronautics 
time of a particle to reach a certain position on a streamline, starting from an initial upstream position. Hence, $f$ satisfies the first-order partial differential equation

$$
\boldsymbol{u}_{0} \cdot \nabla f=c .
$$

Next, an auxiliary source patch grid can be constructed by considering a bundle of streamlines, starting in a upstream rake position and truncated somewhere downstream. Then, $\xi(x, y), \eta(x, y)$ coordinates can be introduced, where a coordinate value $\eta=$ const. is related to a certain streamline. The coordinate $\eta$ can be introduced, e.g., by sequentially numbering the streamlines. The $\xi$ coordinates can be determined by constructing mesh lines orthogonal to the streamlines, e.g., by an advancing front algorithm. Fig. 1 shows

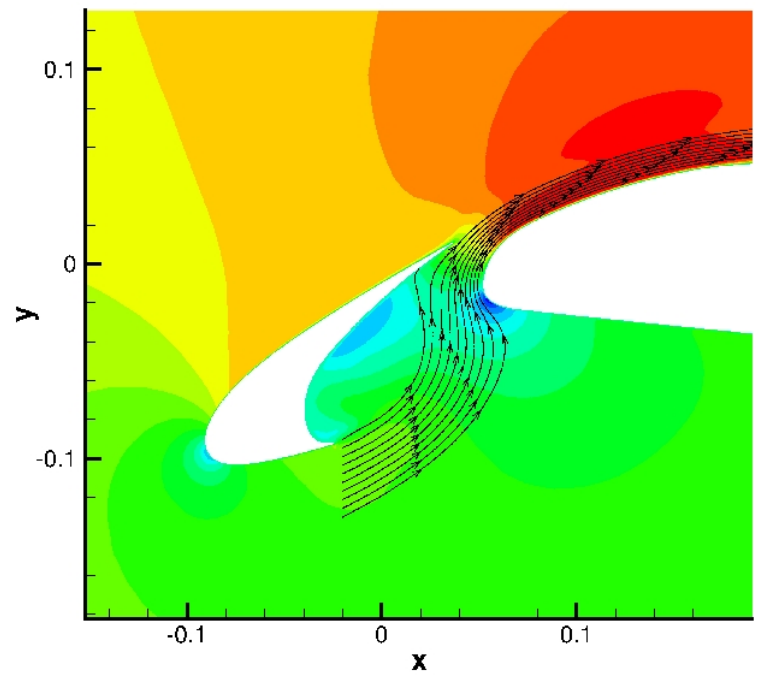

(a) Streamlines in the slat-cove.

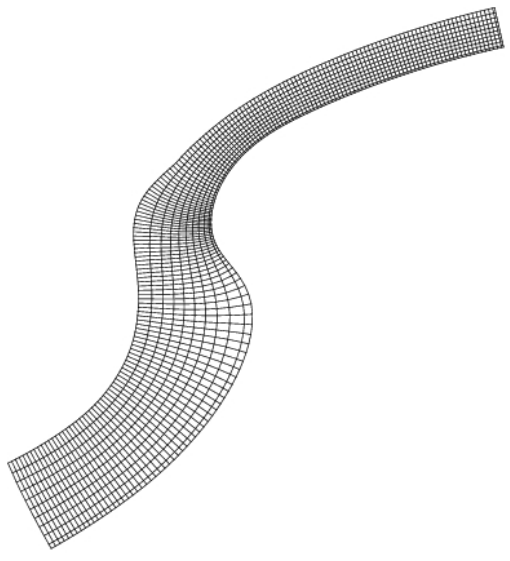

(b) Auxiliary grid.

Figure 1. Streamlines in the slat-cove and auxiliary grid; $\eta=$ const. lines are identical with the streamlines, $\xi=$ const. lines are normal to the streamlines.

a bundle of streamlines in the vicinity of a deployed slat and a related source patch grid. Then, taking into account that the variable $f$ can be expressed as a function of $\xi$ and $\eta$, the convecting white noise field can be written as $\mathcal{U}(f(\xi, \eta)-c t, \eta)$. It is easy to show that this ansatz realizes the convection property of the white-noise field, i.e.,

$$
\frac{\partial \mathcal{U}}{\partial t}+\boldsymbol{u}_{0} \nabla \mathcal{U}=0
$$

where $\boldsymbol{u}_{0}$ denotes the mean-flow field. For this, one has to consider that $f$ satisfies Eq. (19) and $\boldsymbol{\nabla} \eta$ to be orthogonal to the streamline, i.e., $\boldsymbol{u}_{0} \boldsymbol{\nabla} \eta=0$. Finally, with an surface element defined on the source patch by $\left|\frac{\partial\left(x^{\prime}, y^{\prime}\right)}{\partial(\xi, \eta)}\right|$, the integral Eq. (17) becomes

$$
\psi(\boldsymbol{x}, t)=\int_{\xi} \int_{\eta} G\left(\left|\boldsymbol{x}-\boldsymbol{x}^{\prime}(\xi, \eta)\right|\right) \mathcal{U}(f(\xi, \eta)-c t, \eta)\left|\frac{\partial\left(x^{\prime}, y^{\prime}\right)}{\partial(\xi, \eta)}\right| d \xi d \eta
$$

The streamfunction approach does not only realize a perfect solenoidal velocity field but furthermore solves the third discussed problem of the filtered velocity field to exhibit unphysical spherical symmetry in the two-point correlations. Physically, in case of isotropic homogeneous turbulence, the correlation tensor of 
the velocity components $u_{i}$ and $u_{j}$ (with $u_{1}=u, u_{2}=v$ and $u_{3}=w$ ) is ${ }^{5}$

$$
R_{i j}^{0}(r, \tau=0)=[f(r)-g(r)] n_{i} n_{j}+g(r) \delta_{i j},
$$

where $\delta_{i j}$ is the Kronecker symbol, $r=\left|\boldsymbol{r}_{1}-\boldsymbol{r}_{2}\right|$, and $\tau=\left|t_{1}-t_{2}\right|$. The vector components $n_{i}$ stand for the unity vector in the $\boldsymbol{r}_{1}-\boldsymbol{r}_{2}$ direction. For example, in the coordinate direction $x_{1}$ the correlation of $u_{1}, R_{11}^{0}$, becomes $f(r)$, in direction $x_{2}$ it is $g(r)$. Continuity in 3D connects the lateral and longitudinal correlation functions $g(r)$ and $f(r)$, respectively, via ${ }^{5}$

$$
g(r)=f(r)+\frac{r}{2} \frac{d f(r)}{d r} .
$$

In $2 \mathrm{D}$ this relation becomes

$$
g(r)=f(r)+r \frac{d f(r)}{d r} .
$$

As was shown by Careta et al., ${ }^{7}$ a fluctuating streamfunction $\psi$ with spherical symmetric correlation $C(r)$ exactly recovers Eqs. (21) and (23), where the longitudinal and lateral correlation functions of the velocity components $u_{1}=\partial \psi / \partial y, u_{2}=-\partial \psi / \partial x$, are related to the correlation $C(r)$ via

$$
f(r)=-\frac{C^{\prime}(r)}{r}, \quad g(r)=-C^{\prime \prime}(r) .
$$

The prime indicates differentiation with respect to $r$. Hence, using the Gaussian filter kernel based correlation Eq. (11) to express $C(r)$, the longitudinal correlation function becomes

$$
f(r) \propto \exp \left(-\frac{\pi}{4} \frac{x^{2}}{\Delta^{2}}\right) .
$$

In other words, in the streamfunction approach the meaning of $\Delta$ is still to be understood as an integral length scale (that of the longitudinal correlation function $\mathrm{f}(\mathrm{r})$ ). Evaluation of $g(r)$ shows that it approaches negative values for a certain range of $r$.

A $3 \mathrm{D}$ extension of the streamfunction approach is possible by determing the $3 \mathrm{D}$ vector streamfunction $\psi$ through

$$
\boldsymbol{\psi}(\boldsymbol{x}, t)=\int_{\boldsymbol{x}^{\prime}} G\left(\left|\boldsymbol{x}-\boldsymbol{x}^{\prime}\right|, \Delta\left(\boldsymbol{x}^{\prime}\right)\right)\left(\begin{array}{c}
\mathcal{U}_{1}\left(\boldsymbol{x}^{\prime}\right) \\
\mathcal{U}_{2}\left(\boldsymbol{x}^{\prime}\right) \\
\mathcal{U}_{3}\left(\boldsymbol{x}^{\prime}\right)
\end{array}\right) d \boldsymbol{x}^{\prime} .
$$

Here the $\mathcal{U}_{i}$ denote three independent stochastic white-noise fields of the previously explained type and $G$ is a 3D filter kernel. It will be shown elsewhere that the solenoidal 3D velocity field $\boldsymbol{u}=\boldsymbol{\nabla} \times \boldsymbol{\psi}$ satisfies Eq. (21) in conjunction with Eq. (22). However, each component of the vector streamfunction is directly related to the components of the vorticity vector through $\boldsymbol{\omega}=\nabla^{2} \psi$. For airframe noise problems like wing slat- or trailing edge noise, only the vorticity vector component in spanwise direction, say $x_{3}$, will contribute to the sound generation. By neglecting the other components in Eq. (26) (dropping $\mathcal{U}_{1}$ and $\mathcal{U}_{2}$ ), one recovers the previous $2 \mathrm{D}$ formulation (with $\mathcal{U}_{3}$ identified with $\mathcal{U}$ ), which therefore is sufficient for the application to slat-noise problems.

In this paper no attempt is made to include anisotropy into the model. However, anisotropic turbulence based on the modeled isotropic turbulent velocity field, can be achieved with the transformation proposed by Smirnov et al., ${ }^{3}$ which leaves the velocity field solenoidal.

\section{Discrete realization of the acoustic source computation}

The discretization of Eq. (20) realizes the integral through a finite sum over the source patch elements. For this, a constant time increment $\Delta t$ is introduced, which defines discrete points along each individual 
streamline according to the time a Lagrangian particle needs to float from a downstream rake to the actual position. Since the the maximal time to reach the downstream border of a source patch differs from streamline to streamline, the number of discrete points also varies from streamline to streamline. A random number is related to each discrete point, which is scaled according to Eq. (16) with $\sqrt{\Delta A_{i j}}$, for the $i t h$ point on the $j t h$ streamline. If $\Delta t$ agrees with the time step of the CAA method, which is used to compute the acoustic field, the procedure to compute the streamfunction becomes

- For each time increment shift the unscaled random numbers one position downstream and determine local random values by scaling them with the knew local value of $\sqrt{\Delta A_{i j}}$.

- Update the first upstream position with new random values.

- Filter the random field and interpolate the filtered data onto the CAA grid.

Instead of computing the contribution of all random field points within cut-on distance to a certain field position, the value of a certain random point is distributed into the field. Thereby the local length scale at the random point is used to scale the filter kernel. Furthermore, the local value of the kinetic turbulent energy is used to scale the local amplitude $\hat{A}$ of the filter kernel. The exact quantity of $\hat{A}$ based on the RANS mean-flow field will be given below. The filtered values are directly computed for the relevant CAA grid points.

The method can be deemed to be a particle-incell method (PIC), where stochastic particles are floating along streamlines and are interpolated onto the CAA grid using the filter kernel as locally defined blob-functions. However, the quantity that is interpolated onto the CAA grid is a fluctuating streamfunction.

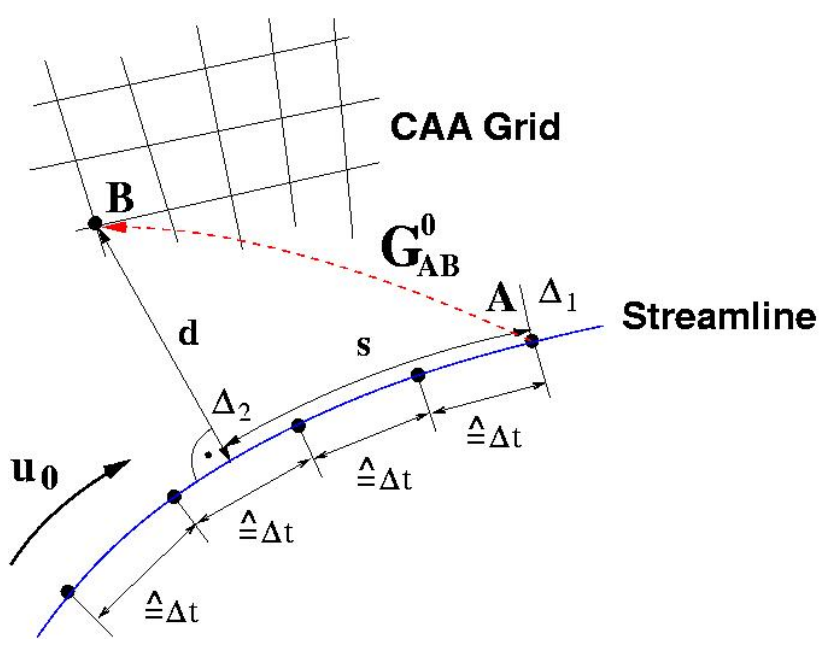

The filter kernel is computed again in a sequence of single-dimensional filter operations, see Fig. 2. First, the random field is filtered along the streamline for each discrete point on the streamline, using the length scale in each random point for the kernel scaling. Next, the intermediate filtered values are distributed onto the CAA grid.

In a preprocessing routine, for each CAA grid point a base point on each streamline is identified, which has the property to mark the smallest distance to the considered grid point for all possible vectors that are normal to the streamline and go through the field point. Usually the base points become placed between discrete points on the streamline. Therefore, 4 -th order interpolation is used to interpolate from the discrete points on the streamline to the base-point. The interpolated local value of the length scale in the base-point is used to compute the decay of the filter-function from the base- to the grid point. If $\Delta_{1}$ denotes the lengthscale at a certain stochastic particle position $\mathrm{A}$ on the streamline, $s$ is the distance along the streamline to the base-point, $\Delta_{2}$ the length-scale in the base-point and $d$ is the distance to the grid point $\mathrm{B}$, Fig. 2, the contribution of the particle to the grid point due to the filter kernel becomes

$$
G_{A B}^{0}:=G^{0}(d, s)=\exp \left(-\frac{\pi}{2} \frac{s^{2}}{\Delta_{1}^{2}}\right) \exp \left(-\frac{\pi}{2} \frac{d^{2}}{\Delta_{2}^{2}}\right) .
$$

For uniform flow with constant length-scale this becomes identical with the Gaussian kernel Eq. (10). For curved streamlines a small distortion of the kernel shape occurs. 
Note that each CAA grid point is maximally linked to each streamline one time, and the number of basepoints is constant and independent of the number of discrete stochastic particle points along the streamline.

Usually, the small CAA time step would demand a too large number of discrete points along the streamline, if for each time increment the random numbers are shifted one full position downstream. In this work a larger spacing along the streamline was allowed by considering random particle positions between discrete points on the streamline. Then the interpolation onto the fixed points along the streamline is realized through the first filter operation, whereby the filter is centered at the local particle position (i.e. it is moved with the random particle), but the filtered values are computed for the fixed streamline points.

\section{Scaling of the filter kernel parameters from RANS}

The filter kernel parameter $\Delta$ and $\hat{A}$ are scaled using the steady RANS computation. In general, from the kinetic turbulent energy $\bar{k}$ and the mean dissipation rate $\epsilon$ an integral length scale can be deduced:

$$
\Delta=c_{l} \frac{\bar{k}^{3 / 2}}{\epsilon} .
$$

Closer scrutinize reveals the constant to be $c_{l} \approx 0.54$. Equating the turbulent viscosity $\nu_{T}$ of the $\bar{k}-\epsilon$ with that of a $\bar{k}-\omega$ model, the relation $\epsilon=C_{\mu} \bar{k} \omega$ follows, where $C_{\mu}=0.09$. Then the length scale in terms of the $\bar{k}-\omega$-model becomes

$$
\Delta=\frac{c_{l}}{C_{\mu}} \frac{\bar{k}^{1 / 2}}{\omega} \approx 6.00 \frac{\bar{k}^{1 / 2}}{\omega} .
$$

The amplitude $\hat{A}$ is scaled by computing from Eq. (17) the value of the kinetic turbulent energy and by relating this expression to the turbulent kinetic energy $\bar{k}$ from the RANS computation. From Eq. (17) the velocity components follow to be

$$
u=\int_{\boldsymbol{x}^{\prime}} \frac{\partial G}{\partial y} \mathcal{U} d \boldsymbol{x}^{\prime}, \quad v=-\int_{\boldsymbol{x}^{\prime}} \frac{\partial G}{\partial x} \mathcal{U} d \boldsymbol{x}^{\prime} .
$$

From Eq. (7), evaluated for $r=0$, the turbulent kinetic energy can be deduced to be

$$
\bar{k}=\frac{\overline{u^{2}}+\overline{v^{2}}}{2}=\frac{1}{2} \int_{\boldsymbol{x}^{\prime}}\left[\left(\frac{\partial G}{\partial x}\right)^{2}+\left(\frac{\partial G}{\partial y}\right)^{2}\right] d \boldsymbol{x}^{\prime} .
$$

Inserting the Gaussian kernel, the amplitude $\hat{A}$ follows to be linked to the value of the turbulent kinetic energy via

$$
\hat{A}=\sqrt{\frac{2}{3 \pi}} \bar{k}^{1 / 2} \approx 0.460 \bar{k}^{1 / 2} .
$$

Note that the amplitude is completely determined by the kinetic turbulent energy, independent of the local value of $\Delta$.

\section{Acoustic simulation techniques}

\section{A. Acoustic Perturbation Equations}

For the acoustic simulations acoustic perturbation equations ${ }^{8}$ (APE) are used, which can be deemed to be a modification of the genuine linearized Euler equations (LEE). The system solved for the pressure and velocity perturbations $\left(p^{\prime}, \boldsymbol{u}^{\prime}\right)$ is

$$
\begin{aligned}
& \frac{\partial p^{\prime}}{\partial t}+c_{0}^{2} \boldsymbol{\nabla} \cdot\left(\rho_{0} \boldsymbol{u}^{\prime}+\boldsymbol{u}_{0} \frac{p^{\prime}}{c_{0}^{2}}\right)=c_{0}^{2} q_{c} \\
& \frac{\partial \boldsymbol{u}^{\prime}}{\partial t}+\boldsymbol{\nabla}\left(\boldsymbol{u}_{0} \cdot \boldsymbol{u}^{\prime}\right)+\nabla\left(\frac{p^{\prime}}{\rho_{0}}\right)=\boldsymbol{q}_{m} .
\end{aligned}
$$


Here the $\rho_{0}, p_{0}$, and $\boldsymbol{u}_{0}$ denote the density, pressure and the velocity of the time averaged flow, respectively. Furthermore, $c_{0}=\sqrt{\gamma p_{0} / \rho_{0}}$ is the local sound speed. The right-hand side sources $q_{c}$ and $\boldsymbol{q}_{m}$ will be defined below. The homogeneous equation system with the sources set to zero can be shown to be equivalent to the convective wave equation for irrotational mean-flows ${ }^{8}$

$$
\mathcal{L} \varphi=\left[\frac{D_{0}}{D t}\left(\frac{1}{c_{0}^{2}} \frac{D_{0}}{D t}\right)-\frac{1}{\rho_{0}} \nabla \cdot\left(\rho_{0} \boldsymbol{\nabla}\right)\right] \varphi=0,
$$

where $D_{0} / D t=\partial / \partial t+\boldsymbol{u}_{0} \cdot \boldsymbol{\nabla}$ denotes the substantial time derivative and $\varphi$ is an acoustic potential that is related to the fluctuating pressure through the expression

$$
p^{\prime}=-\rho_{0} \frac{D_{0} \varphi}{D t} .
$$

Möhring ${ }^{9}$ proved for the wave-operator $\mathcal{L}$ of Eq. (34), that it is stable for arbitrary mean flows. This feature is due to the fact that Eq. (34), unlike other convective wave equations, does not include additional hydrodynamic modes that can become subject to growing hydrodynamic instabilities. It is easy to show that the APE system Eqs. $(32,33)$ also exclude the vortical (hydrodynamical) modes by taking the curl of Eq. (33). Considering the source term on the right-hand side, the vorticity equation of the APE system becomes

$$
\frac{\partial \boldsymbol{\omega}^{\prime}}{\partial t}=\boldsymbol{\nabla} \times \boldsymbol{q}_{m}
$$

Hence, the perturbation vorticity $\boldsymbol{\omega}^{\prime}=\boldsymbol{\nabla} \times \boldsymbol{u}^{\prime}$, on the left-hand side is completely controlled by the righthand side (RHS) source term. For the homogeneous system with all sources removed, the vorticity equation reduces to the statement that the vorticity remains constant (zero), i.e., unlike the LEE, the APE do not support the convective vorticity mode. Due to the number of variables in the Eqs. $(32,33)$, the system supports more eigenmodes than Eq. (34). However, since the vortical mode is degenerated, the APE system and Eq. (34) are completely equivalent, i.e., the system excludes the hydrodynamic instabilities, too. This is an important feature if linear perturbations are forced with sources, since growing instabilities will cause divergent solutions that are neither limited by non-linear saturation nor viscous effects.

In general, the linearized Euler equations can be recast ${ }^{8}$ into an equivalent system based on an acoustic potential and variables for the vorticity mode. The LEE exhibit the vorticity convection mode, but the wave equation of the equivalent LEE system conforms with Eq. (34). However, the equations of the equivalent LEE system are coupled in rotational mean-flows, such that additional acoustic sources will appear on the right-hand side of Eq. (34) due to the interaction of acoustic and vorticity modes, respectively. The effect of these secondary source on wave propagation is neglected if the homogeneous Eq. (34) or the homogeneous system Eqs. $(32,33)$ are applied in rotational flow regions. The homogeneous APE system, nevertheless, resolves convection effects with good accuracy even in mean flows with mild levels of mean vorticity as was demonstrated in Ref. ${ }^{8}$

\section{B. APE based acoustic analogy}

Fig. 3 gives a principle classification of acoustic domains that occur for a body in turbulent flow.

In the near-field the flow is dominated by highly non-linear turbulent flow features that are accurately described by the full set of Navier-Stokes equations (referred to as the Navier-Stokes domain). However, in the far-field the time averaged mean-flow becomes irrotational and constant, and acoustic fluctuations that occur in this region are usually so small that their propagation is exactly described by the homogeneous wave equation for constant flow (referred to as homogeneous acoustic domain).

Lighthill's acoustic analogy ${ }^{10}$ is a recast of the governing flow equations into that type of a simple wave equation on the left-hand side plus additional sources on the right-hand side. Since the homogeneous left-hand side wave equation describes wave propagation exactly in the homogeneous domain (neglecting non-linear terms and considering the constant flow case via Galilean transform of the non-flow wave equation), one

10 of 23

American Institute of Aeronautics and Astronautics 
can conclude that the right-hand side sources of Lighthill's analogy must vanish in that domain. However, approaching the body closer, the mean-flow, although still irrotational, becomes non-uniform. Since the physical correct wave equation in this domain is Eq. (34) that starts to deviate from the constant-flow wave equation, the sources of Lighthill's acoustic analogy will be non-zero in this domain (referred to as inhomogeneous acoustic domain). Note, however, that the homogeneous wave equation, Eq. (34), is still sufficient to describe the acoustics properly, i.e., the analogy source term does not describe 'true' acoustic sources in this domain.

Lighthill's analogy applies the underlying wave equation of the homogeneous acoustic domain with virtual and acoustic sources on the RHS in all three acoustic domains. A straight forward extension of this concept would be to recast the governing flow equations such that the left-hand side realizes the wave equation of the inhomogeneous domain plus the remaining sources on the right-hand side. Hence, wave propagation is computed in all three domains by the convective wave equation that governs the complete nonuniform and irrotational flow

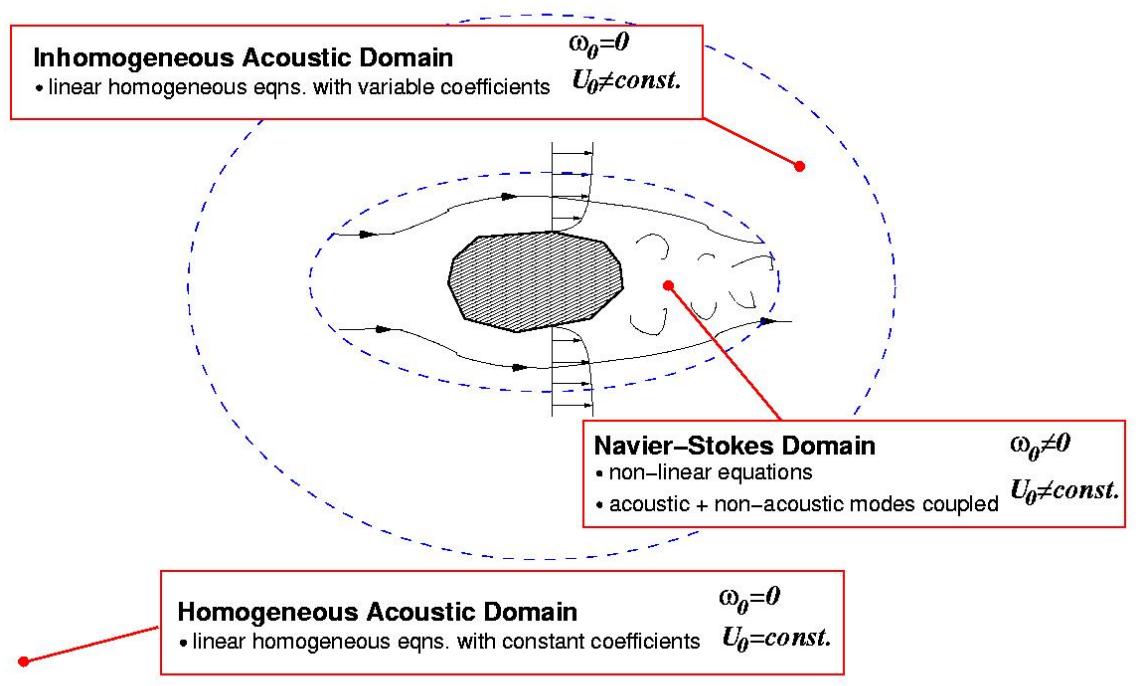
field (homogeneous and inhomogeneous domain). Such an extension would have the advantage that the region where the sources are non-zero re-

Figure 3. Domains of CAA. duces to the Navier-Stokes domain where vorticity is apparent.

Since the homogeneous APE system realizes an exact solution of the convective wave equation, recasting the governing flow equations such that the left hand side equals the system Eqs. $(32,33)$, an extended analogy can be found, which is based on a set of dependent variables, namely $p^{\prime}$ and $\boldsymbol{u}^{\prime}$, rather than on one scalar variable. Similar to the previous discussion, it can be concluded, that the such defined vortex sound sources on the RHS will vanish in and beyond the inhomogeneous acoustic domain, respectively. Note that an acoustic analogy concept, defined in the aforementioned way, must not necessarily be based on one scalar wave equation, nor is it necessary that it can be solved via integral methods.

Starting from the Navier-Stokes equations in non-linear disturbance formulation, the source terms of an APE based analogy become ${ }^{8,11}$ (neglecting non-linear perturbation entropy terms)

$$
\begin{aligned}
& q_{c}=-\boldsymbol{\nabla} \cdot\left(\rho^{\prime} \boldsymbol{u}^{\prime}\right)^{\prime}+\frac{\rho_{0}}{c_{p}} \frac{D_{0} s^{\prime}}{D t} \\
& \boldsymbol{q}_{m}=-(\boldsymbol{\omega} \times \boldsymbol{u})^{\prime}+T^{\prime} \boldsymbol{\nabla} s_{0}-s^{\prime} \boldsymbol{\nabla} T_{0}-\left(\boldsymbol{\nabla} \frac{\left(u^{\prime}\right)^{2}}{2}\right)^{\prime}+\left(\frac{\boldsymbol{\nabla} \cdot \underline{\tau}}{\rho}\right)^{\prime},
\end{aligned}
$$

where $(\ldots)^{\prime}:=(\ldots)-\overline{(\ldots)}$ denotes perturbations of terms obtained by subtracting from an actual term its time average, indicated by an overline. Major vortex source term is the fluctuating Lamb vector

$$
\boldsymbol{q}_{m}=-(\boldsymbol{\omega} \times \boldsymbol{u})^{\prime}=-\boldsymbol{\omega}_{0} \times \boldsymbol{u}^{\prime}-\boldsymbol{\omega}^{\prime} \times \boldsymbol{u}_{0}-\left(\boldsymbol{\omega}^{\prime} \times \boldsymbol{u}^{\prime}\right)^{\prime} .
$$


A similar vortex source term appears in the acoustic analogies of Powell, Howe, and Möhring. ${ }^{12,13,9}$ The extension of the acoustic analogy concept from a scalar wave equation to a set of linearized inhomogeneous equations was recently also discussed by Goldstein. ${ }^{14}$ Note that the occurrence of vorticity in the vortex source acknowledges the previously made assumption that the sources will vanish in the inhomogeneous acoustic domain, where the flow is irrotational.

\section{Numerical Implementation}

The computations in this paper are carried out with DLR's CAA code PIANO. The computational problem to solve for perturbation variables $\boldsymbol{U}=\left(\rho^{\prime}, \boldsymbol{u}^{\prime}, p^{\prime}\right)^{T}$ over a mean flow field $\left(\rho_{0}, \boldsymbol{u}_{0}, p_{0}\right)^{T}$ formally looks like

$$
\frac{\partial \boldsymbol{U}}{\partial t}+\boldsymbol{A}_{0 i} \frac{\partial \boldsymbol{U}}{\partial x_{i}}+\boldsymbol{H}_{0} \boldsymbol{U}=\boldsymbol{S}
$$

In Eq. (40) Einstein's summation condition holds, i.e., products of terms with equal indices are summed over all spatial directions, and a source vector $\boldsymbol{S}$ appears on the right-hand side. For the acoustic perturbation equations, Eqs. (32) and (33), the matrices and source terms become in 2D

$\boldsymbol{A}_{0 i}=\left(\begin{array}{cccc}u_{0 i} & \rho_{0} \delta_{1 i} & \rho_{0} \delta_{2 i} & 0 \\ 0 & u_{01} \delta_{1 i} & u_{02} \delta_{1 i} & \delta_{1 i} / \rho_{0} \\ 0 & u_{01} \delta_{2 i} & u_{02} \delta_{2 i} & \delta_{2 i} / \rho_{0} \\ 0 & \gamma p_{0} \delta_{1 i} & \gamma p_{0} \delta_{2 i} & u_{0 i}\end{array}\right), \quad \boldsymbol{H}_{0}=\left(\begin{array}{cccc}\frac{\partial u_{0 i}}{\partial x_{i}} & \frac{\partial \rho_{0}}{\partial x_{1}} & \frac{\partial \rho_{0}}{\partial x_{2}} & 0 \\ 0 & \frac{\partial u_{01}}{\partial x_{1}} & \frac{\partial u_{02}}{\partial x_{1}} & -\frac{1}{\rho_{0}^{2}} \frac{\partial \rho_{0}}{\partial x_{1}} \\ 0 & \frac{\partial u_{01}}{\partial x_{2}} & \frac{\partial u_{02}}{\partial x_{2}} & -\frac{1}{\rho_{0}^{2}} \frac{\partial \rho_{0}}{\partial x_{2}} \\ 0 & c_{0}^{2} \frac{\partial \rho_{0}}{\partial x_{1}} & c_{0}^{2} \frac{\partial \rho_{0}}{\partial x_{2}} & c_{0}^{2} \frac{\partial}{\partial x_{i}}\left(\frac{u_{0 i}}{c_{0}^{2}}\right.\end{array}\right), \quad \boldsymbol{S}=\left(\begin{array}{c}q_{c} \\ \boldsymbol{q}_{m} \\ c_{0}^{2} q_{c}\end{array}\right)$

where $\delta_{i j}$ is the Kronecker symbol with $\delta_{i j}=0$ for $i \neq j$ and $\delta_{i j}=1$ else, and $\gamma$ is the isentropic exponent. The components of the source vector $\boldsymbol{S}$ are defined by Eqs. (37) and (38). Note that the governing equation for the density is decoupled from the remaining part such that the first equation could be dropped. The spatial discretization is accomplished with the fourth-oder dispersion relation preserving (DRP) scheme of Tam \& Webb. ${ }^{15}$ For the use on curvilinear multi-block grids Eq. (40) is extended with a metric, where the metric terms are computed consistently with the DRP scheme, too. At far-field boundaries the asymptotic radiation and outflow boundary condition of Tam \& $\mathrm{Webb}^{15}$ is used. The solid wall boundary condition is implemented based on a ghost point beneath the surface that yields the proper surface wall normal pressure gradient. ${ }^{16}$ To suppress high frequency spurious waves artificial selective damping according to Tam \& Dong ${ }^{17}$ has been used. The time integration is carried out with a fourth-order Runge Kutta method.

\section{Evaluation of the source terms from digital filtering}

For the acoustic computations of vortex sound just the vorticity term, Eq. (39), is considered. As was shown in Ref., ${ }^{18}$ the sound generation at a solid surface is determined by the vortex induced wall normal solenoidal velocity components. The kinematic boundary condition demands that component to be canceled by an opposite wall normal irrotational velocity component, that will give rise to acoustic radiation, see e.g. pp. 220-222 in Ref. ${ }^{19}$ Hence, the sound sources at solid surfaces are linearly dependent on the fluctuation velocities, i.e. establish linear acoustic sources. The non-linear sources are important if the turbulence related noise generation is considered, i.e., for jet noise problems. Note that even in Lilley's equation, incorporating solid surfaces via generalized functions, linear sources occur on the RHS, that describe sound generation due to solid surfaces. The idea of the wall-normal solenoidal velocity component to be the major source term for vortex-surface interaction noise agrees with the concept of an 'incompressible upwash velocity' introduced by Howe in Ref. ${ }^{20}$

The solenoidal digital filtering procedure provides the value of the streamfunction, Eq. (20) and, via 4th order numerical differentiation, its time derivative on the CAA grid. The velocity components and their 
derivatives are found, computing the gradient of the streamfunction and its time derivative, respectively, with the DRP scheme on the CAA grid. The fluctuating vorticity could also be obtained by one further spatial differentiation step. However, to avoid the additional overhead that would become necessary due to the additional multi-block exchange, an alternative source term is considered such that only the fluctuating velocities and their time derivative have to be known. It was shown in Ref. ${ }^{18}$ for a trailing edge problem that the sound generation of the turbulent flow field is negligible compared to the surface source contribution. The fluctuating Lamb vector can be split into a irrotational and an solenoidal part. The solenoidal part is the only contributor to vorticity in the APE. Taking the curl of Eq. (33) with source Eq. (39) yields the fluctuating vorticity, induced by this part of the source term, see Eq. (36).

Now, the vorticity should be equal to that of the prescribed solenoidal velocity field $\boldsymbol{u}_{\psi}^{\prime}$. It is easy to show, that a source

$$
\boldsymbol{q}_{m}=\frac{\partial \boldsymbol{u}_{\psi}^{\prime}}{\partial t}
$$

satisfies this condition by inserting it into Eq. (36), i.e., the RHS represents the solenoidal part of $\boldsymbol{q}_{m}$. The time-derivative of the stochastically modeled velocity field has been used as source term for the slat-noise computations.

\section{Computational Results}

\section{A. Test driver runs: homogeneous isotropic turbulence}

As a first test-case the stochastic realization of isotropic turbulence with the solenoidal digital filtering method has been considered. Fig. 4(c) shows a snapshot of the $\omega_{3}$ vorticity component of the fluctuating pseudoturbulent field. The mean-flow is uniform and running from left to right. From this fluctuating velocity field the tensor components of Eq. (21), $R_{i j}^{0}(r, \tau)$, are processed. For this, no temporal separation is considered, i.e., $\tau=0$ in Eq. (21). The quantity $r$ denotes the relative distance to a considered point, $r=\sqrt{\Delta x^{2}+\Delta y^{2}}$. The normal vector components in Eq. (21) become $n_{1}=\Delta x / r$, and $n_{2}=\Delta y / r$. Hence, each component $R_{i j}^{0}(r, 0)$ of the correlation tensor is a field function. The Figs. 4 and 5 juxtapose the analytical solution to the stochastically generated result. Almost perfect agreement is obtained. The analytical solution is based on a longitudinal correlation $f(r):=\exp \left(-\frac{\pi}{4} \frac{x^{2}}{L^{2}}\right)$ and its related lateral correlation $g(r)=f+f^{\prime} / r$, Eq. (23), whereby the filter kernel is set to

$$
G^{0}(x)=\exp \left(-\frac{\pi}{2} \frac{x^{2}}{L^{2}}\right)
$$

$L$ denotes the integral length-scale of the longitudinal correlation function used for the problem.

Fig. 6(a) depicts the correlations along a horizontal cut $\Delta y=0$. The black lines indicate the target distributions for the longitudinal correlation function $f$, the $2 \mathrm{D}$ lateral correlation function $g$ and the theoretical correlation of the vorticity $\omega_{3}=\partial v / \partial x-\partial u / \partial y$. The colored triangles represent the respective solutions from the post-processing of the pseudo turbulence. Again almost perfect quantitative agreement is achieved. Fig. 6(b) shows computational results also including a time-scale according to Section IIA into the model. The plot shows the longitudinal correlations for various time separations $\tau$. The black lines indicate the target functions for the spatial and temporal correlations. Very good agreement is achieved between the target functions and the stochastic representation.

\section{B. Slat-noise simulations}

Fig. 7 shows the CAA grid used for the slat-noise computations. It consists of 25 blocks with about $250 \mathrm{k}$ mesh points. It resolves the acoustic field in a $5 c \times 5 c$ box, where $c$ denotes the main-element chord length. Based on a dimensional chord length $c=0.4 \mathrm{~m}$, the grid is sufficient to resolve frequencies up to $12 \mathrm{kHz}$. The

13 of 23

American Institute of Aeronautics and Astronautics 
topology is of a mixed $H / C$-type. An $H$-topology is used in the outer region, which yields almost Cartesian cells in the far-field. A $C$-grid is used in the vicinity of the airfoil to resolve the airfoil with the slat. The mesh resolution is enhanced in the slat-cove to resolve the estimated length scale of the source term properly.

The RANS mean-flow field is computed with DLR's unstructured flow-solver TAU. The free flow Mach number, which is considered in this paper is $M=0.10$. Furthermore, a Menter SST turbulence model ${ }^{21}$ with and without Kato-Launder modification, ${ }^{22}$ respectively, is used. Fig. 8 depicts the distribution of turbulent kinetic energy in the slat-cove region. The solution with Kato-Launder modification, ${ }^{22}$ Fig. 8(a), exhibits a physically meaningful solution, whereas the Menter SST model yields an artificial turbulent kinetic energy spot at the main element nose, which has one order of magnitude higher levels than the Kato-Launder solution. Since $\bar{k}$ directly determines the strength of the acoustic source terms via Eq. (30), the artifical $\bar{k}$-spot will probably yield an overamplified acoustic response. However, Fig. 9 depicts the integral length scale distribution due to Eq. (29) for both models with $c_{l} / C_{\mu}=6.0$, which yield the same magnitudes in the slat-cove region. The black circles in Fig. 9 indicate the size of the length-scale $\Delta$, where the radius of the circles is equal to $\Delta$.

Fig. 10 shows a snapshot of the $v$-velocity field, generated from the solenoidal digital filtering (SDF) procedure, utilizing the RANS solutions of $\bar{k}$ and the length scale from the Kato-Launder model. A bundle of 20 streamlines is considered for the source computation. Their extension in the computational domain is shown in Fig. 1. In the mean, about 800 discrete points along the streamlines are used, corresponding to a time increment from point to point of $\Delta t=4 \cdot 10^{-3}$. The used method has a memory overhead of about $20 \mathrm{MB}$, compared to $700 \mathrm{MB}$ memory requirement for the baseline CAA solver without source model. In principle it would be possible to incorporate also the recirculation bubble in the slat-cove into the source model by extending the model to closed loops of streamlines. However, it is believed that the major source of slat-noise is due to the vortical structures that are generated in the slat-cove shear-layer and that convect past the slat trailing edge. The scaling factors are $c_{l} / C_{\mu}=2.0$ in Fig. 10(a) and $c_{l} / C_{\mu}=6.0$ in Fig. 10(b), respectively. Both results of the pseudo-turbulent flow field exhibit similar details: from the slat hook the flow structures emerge, as one would expect it from growing instabilities in a shear layer. However, note that this solution is a complete reconstruction from a steady state RANS solution.

Fig. 11 compares the contour levels of the turbulent kinetic energy with that reconstructed from SDF, using the RANS reference solution to scale the filter parameters. A qualitatively good agreement in the field distribution of the target and the stochastically induced levels is visible.

Fig. 12 shows two snapshots of the unsteady acoustic pressure field that is generated due to the SDF source model. Fig. 12(a) presents a solution for the turbulence model with Kato-Launder modification, Fig. 12(b) for the Menter SST baseline case. Each computation took about 5CPUh on a NEC-SX6 computer. Both acoustic fields exhibit the same directivities. The acoustic field roughly corresponds to that one would expect from a dipole source placed at the slat trailing edge with its axis normal to the slat chord. Also the apparent wave-lengths have similar magnitudes. This might be due to the fact that both turbulence models reproduce the same length-scales in the slat-region.

Fig. 12(c) depicts narrow band spectra for a point $1.5 \mathrm{c}$ below the slat trailing edge for both turbulence models. The spectra are related to a dimensional frequency, which corresponds to a model with $0.4 \mathrm{~m}$ dimensional chord length. As expected, due to the excessive artificial $\bar{k}$-spot at the leading edge, the Menter SST baseline model clearly overpredicts the soundpressure levels. The difference in the sound pressure levels is about $13 \mathrm{~dB}$, which conforms to the difference in the $\bar{k}$-distributions that exceeds one order of magnitude. However, the decay of the spectrum is predicted similarly by both models. The narrow band decay is confined to a range between $f^{-2}$ and $f^{-3}$, which roughly corresponds to the 12 th octave decay between $f^{-1}$ and $f^{-2}$ that was found by Choudhari et al. ${ }^{23}$ in measurements.

Finally, Fig. 13 compares directivities for both models for four different frequencies. The directivities are computed for a circle with center at the slat trailing edge and radius 1.5c. Similar shapes are obtained for both underlying turbulence models. For the lowest frequency of $1 \mathrm{kHz}$, Fig. 13(a), the directivity in the upper half plane exhibits a directivity pattern, which is well known for trailing edge noise, ${ }^{11}$ however mirrored at a vertical axis. In other words, it agrees with the directivity of an edge noise source located at 
the leading edge of a thin profile. Obviously, for the small frequencies the directivity is dominated by the scattering of the acoustic waves at the main-element. At the highest frequency, Fig. 13(d), the directivity shows again a typical trailing edge pattern. But in this frequency range, the pattern is rotated such that the usual upstream direction points to the ground. The direction roughly agrees with the slat chord angle. Hence, for the higher frequency range, the directivity is mostly affected by the slat geometry.

\section{Conclusion}

The stochastic method presented in this paper is capable to generate a pseudo-turbulent velocity field, which exactly reproduces the second-order two-point correlation tensor of homogeneous isotropic turbulence in two or three dimensions, respectively. It is time- and memory efficient, strictly solenoidal, and can easily be applied to the highly non-uniform mean-flow field in the slat-cove region using local results for the kinetic turbulent energy and the corresponding turbulent length scale from the RANS solution. Furthermore, it avoids the occurrence of shear decorrelations and resolves broadband spectra continuously. A further extension to non-homogeneous anisotropic solenoidal flows is possible with the transformation proposed by Smirnov et al. ${ }^{3}$ First computations reveal, that one has to care about the turbulence model used for the RANS mean-flow computations. Further work will focus on Reynold stress turbulence models.

\section{References}

\footnotetext{
${ }^{1}$ Dobrzynski, W. and Pott-Pollenske, M., "Slat Noise Studies for Farfield Noise Prediction," 2001, AIAA Pap. 2001-2158.

${ }^{2}$ Singer, B., Lockard, D., and Brentner, K., "Computational Aeroacoustics Analysis of Slat Trailing-Edge Flow," AIAA Journal, Vol. 38(9), 2000.

${ }^{3}$ Smirnov, A., Shi, S., and Celik, I., "Random Flow Generation Technique for Large Eddy Simulations and Particle Dynamics Modeling," Journal of Fluids Engineering, Vol. 123, 2001, pp. 359-371.

${ }^{4}$ Khavaran, A. and Bridges, J., "Modelling of fine-scale turbulence mixing noise," Journal of Sound and Vibration, Vol. 279, 2005, pp. 1131-1154.

${ }^{5}$ Batchelor, G., The Theory of Homogeneous Turbulence, Cambridge University Press, 1960.

${ }^{6}$ Klein, M., Sadiki, A., and Janicka, J., "A digital filter based generation of inflow data for spatially developing direct numerical or large eddy simulations," J. Comp. Phys., Vol. 186, 2003, pp. 652-665.

${ }^{7}$ Careta, A., Sagués, F., and Sancho, J., "Stochastic generation of homogeneous isotropic turbulence with well-defined spectra," Physical Review E, Vol. 48(3), 1993, pp. 2279-2287.

${ }^{8}$ Ewert, R. and Schröder, W., "Acoustic Perturbation Equations Based on Flow Decomposition via Source Filtering," J. Comput. Phys., Vol. 188, 2003, pp. 365-398.

${ }^{9}$ Möhring, W., "Modelling Low Mach Number Noise," Mechanics of Sound Generation in Flows, edited by E.-A. Müller, Springer, 1979.

${ }^{10}$ Lighthill, M. J., "On Sound Generated Aerodynamically: I. General Theory," Proc. R. Soc. London Ser. A, Vol. 211, 1952 , pp. 564-587.

${ }^{11}$ Ewert, R. and Schröder, W., "On the simulation of trailing edge noise with a hybrid LES/APE method," Journal of Sound and Vibration, Vol. 270, 2004, pp. 509-524.

${ }^{12}$ Powell, A., "Theory of vortex sound," Journal of the acoustical society of america, Vol. 36/1, 1964, pp. 177-195.

${ }^{13}$ Howe, M. S., "Contributions to the Theory of Aerodynamic Sound, with Application to Excess Jet Noise and the Theory of the Flute," J. Fluid Mech., Vol. 71, No. 4, 1975, pp. $625-673$.

${ }^{14}$ Goldstein, M., "A generalized acoustic analogy," J. Fluid Mech., Vol. 488, 2003, pp. 315-333.

${ }^{15}$ Tam, C. and Webb, J., "Dispersion-Relation-Preserving Finite Difference Schemes for Computational Acoustics," J. Comp. Phys., Vol. 107, 1993, pp. 262-281.

${ }^{16}$ Tam, C. and Dong, Z., "Wall boundary condition for high-order finite-difference schemes in computational aeroacoustics," Theoret. Comput. Fluid Dynamics, Vol. Vol. 6, No. 6, 1994, pp. 303-322.

${ }^{17}$ Tam, C. and Dong, Z., "A Study of the Short Wave Components in Computational Acoustics," Vol. 107, 1993, pp. 262281.

${ }^{18}$ Ewert, R. and Bauer, M., "Towards the prediction of broadband trailing edge noise via stochastic surface sources," 2004, AIAA Pap. 2004-2861.

${ }^{19}$ Goldstein, M., Aeroacoustics, McGraw-Hill, 1976.

${ }^{20}$ Howe, M., "Trailing Edge Noise At Low Mach Numbers," Journal of Sound and Vibration, Vol. 225(2), 1999, pp. 211-238.

${ }^{21}$ Menter, F., "Zonal Two Equation k- $\omega$ Turbulence Models for Aerodynamic Flows," 1993, AIAA Pap. 93-2906.
}

15 of 23 


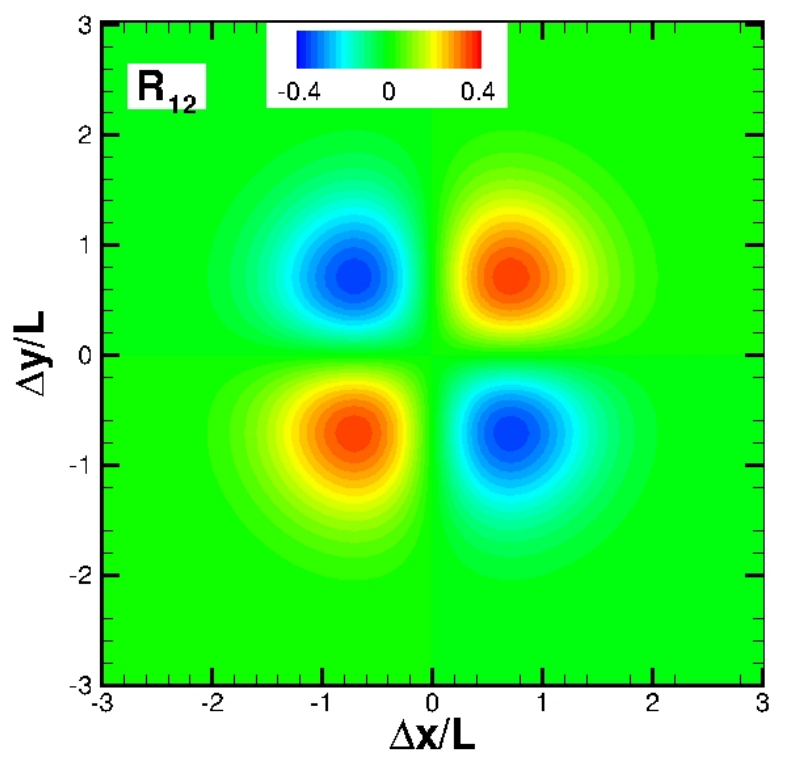

(a) $R_{12}^{0}(\Delta x, \Delta y)$ analytical, Eq. (21) with $f(r):=$ $\exp \left(-\frac{\pi}{4} \frac{x^{2}}{L^{2}}\right)$.

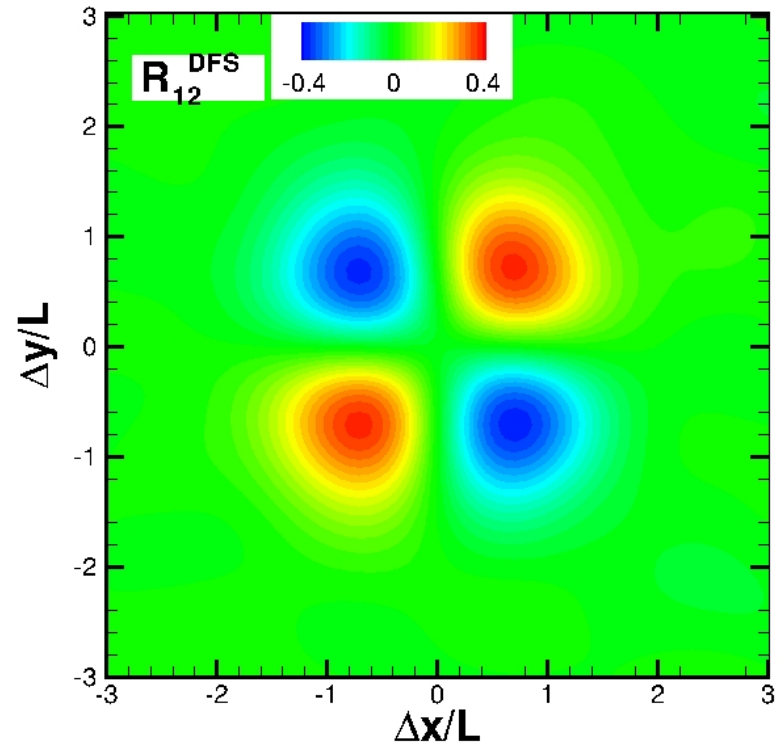

(b) $R_{12}^{0}$ from stochastic simulation.

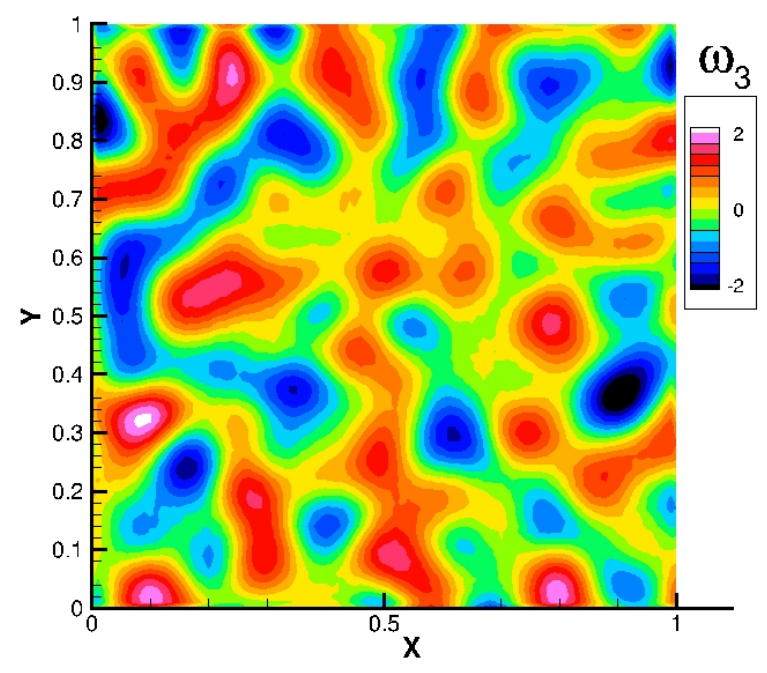

(c) Snapshot of $\omega_{3}$-vorticity.

Figure 4. Stochastic realization of homogeneous isotropic turbulence via solenoidal digital filtering, I. 


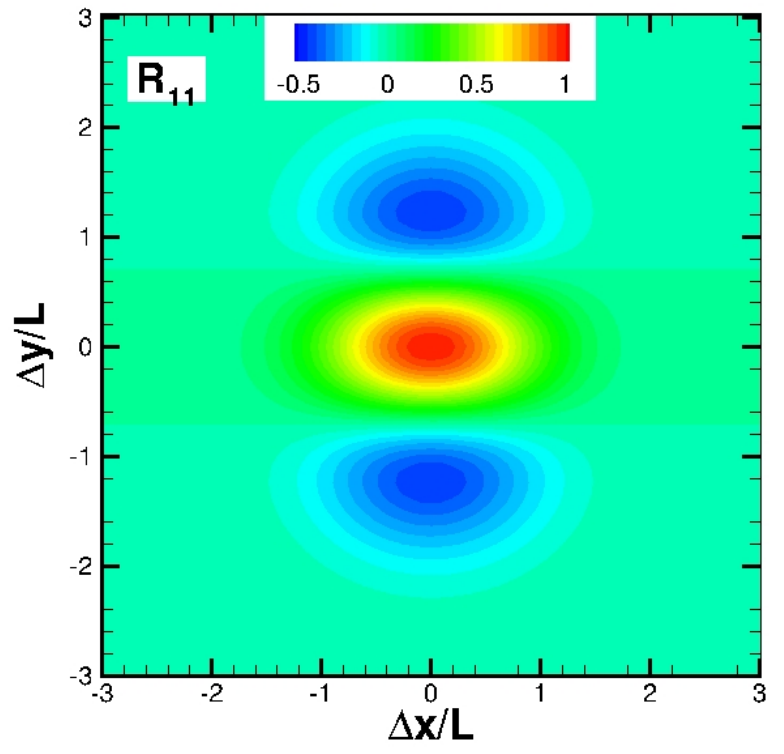

(a) $R_{11}^{0}(\Delta x, \Delta y)$ analytical, Eq. (21) with $f(r):=$ $\exp \left(-\frac{\pi}{4} \frac{x^{2}}{L^{2}}\right)$.

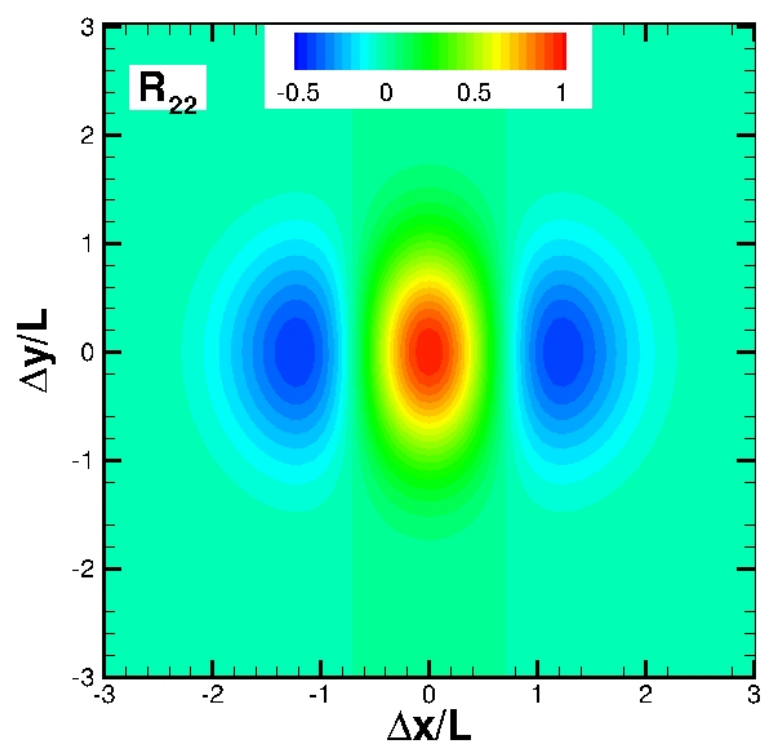

(c) $R_{22}^{0}(\Delta x, \Delta y)$ analytical, Eq. (21) with $f(r)=$ $\exp \left(-\frac{\pi}{4} \frac{x^{2}}{L^{2}}\right)$.

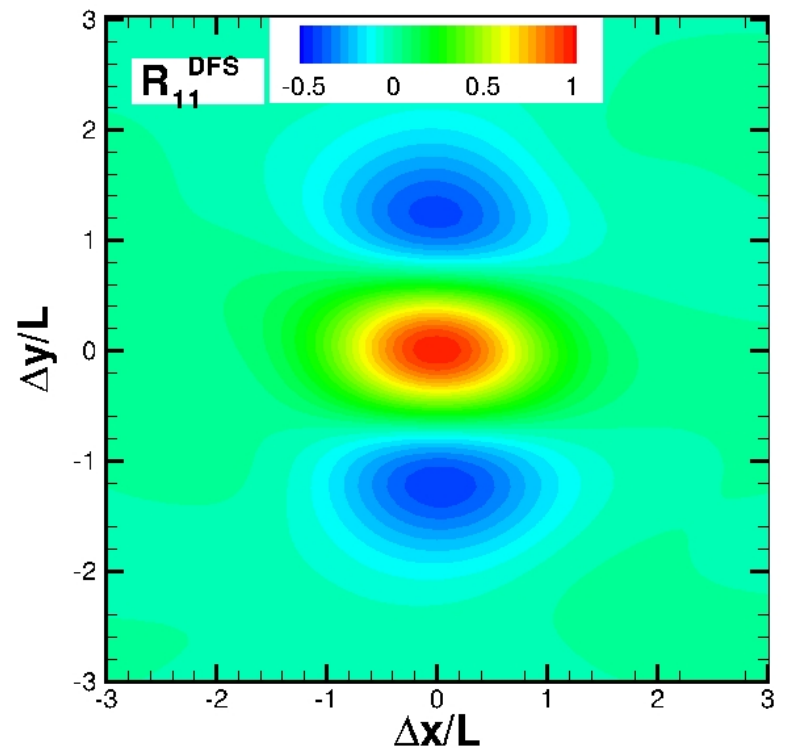

(b) $R_{11}^{0}$ from stochastic simulation.

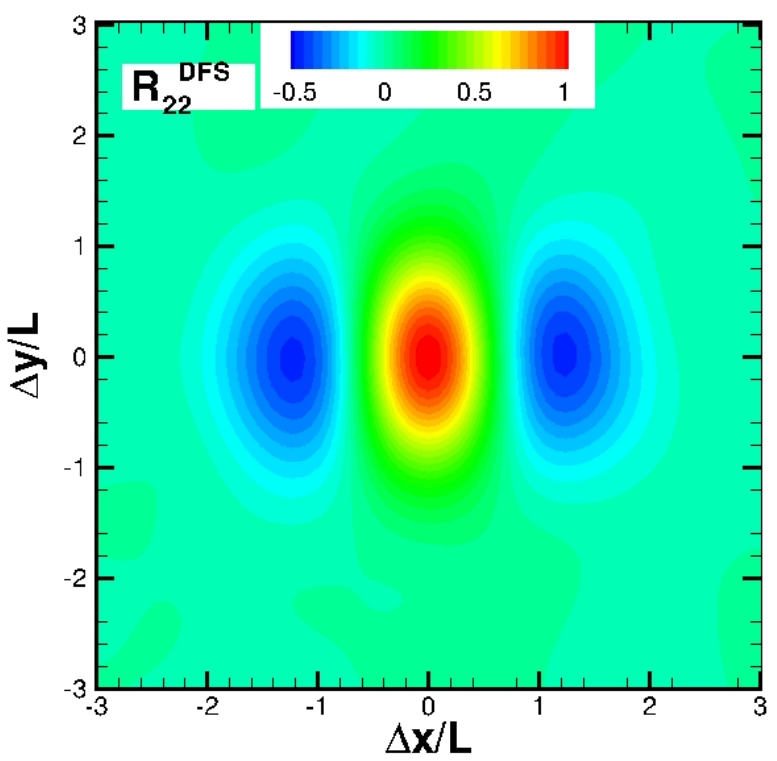

(d) $R_{22}^{0}$ from stochastic simulation.

Figure 5. Stochastic realization of homogeneous isotropic turbulence via solenoidal digital filtering, II. 


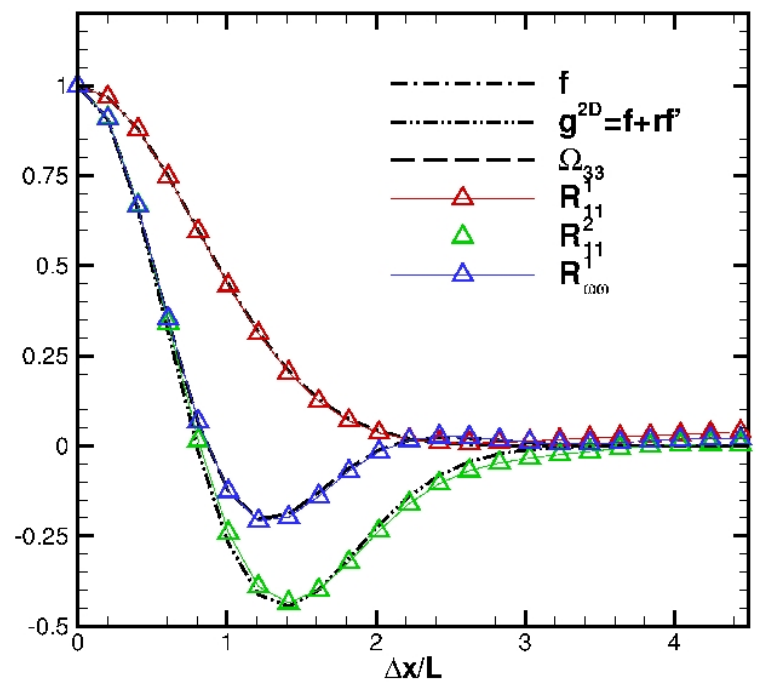

(a) Spatial two-point correlations; black line: target function, colored triangles: reconstruction.

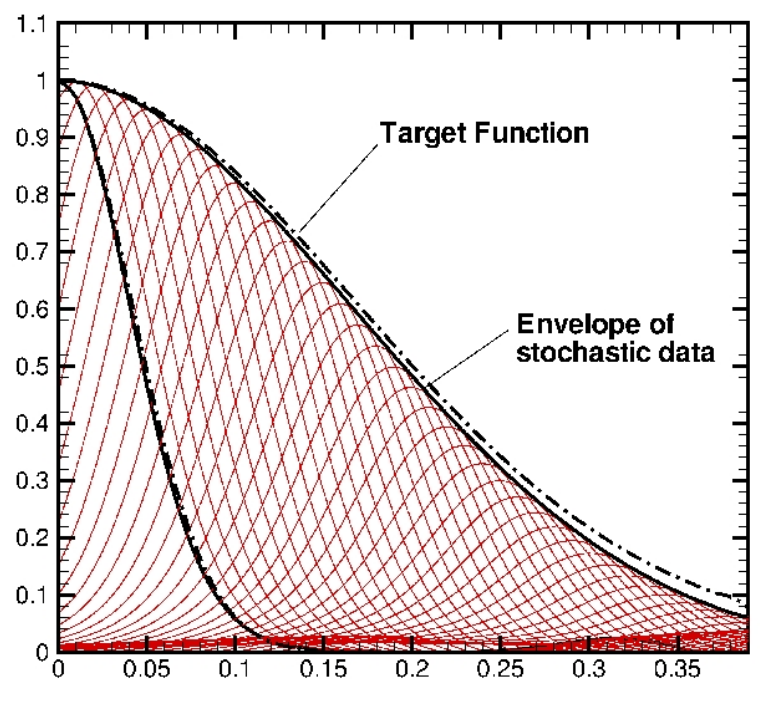

(b) Two-point space-time correlations over separation distance $\Delta x$ for different time differences $\Delta t$.

Figure 6. Reconstruction of spatial and temporal-spatial two-point correlations from stochastically generated homogeneous isotropic turbulence.

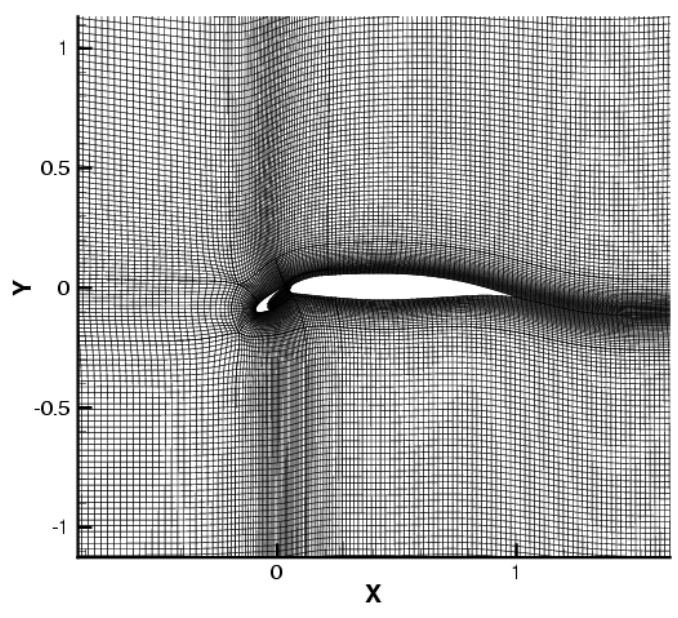

(a) Full domain.

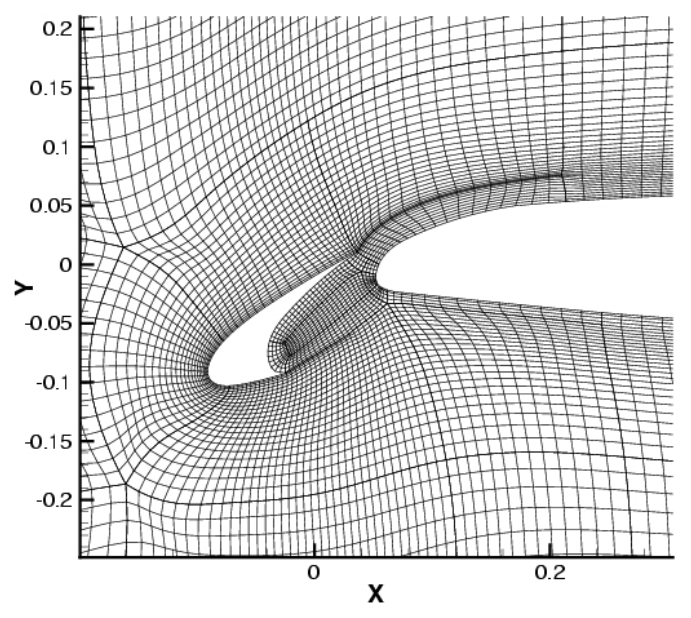

(b) Slat cove.

Figure 7. CAA grid, every other grid line shown. 


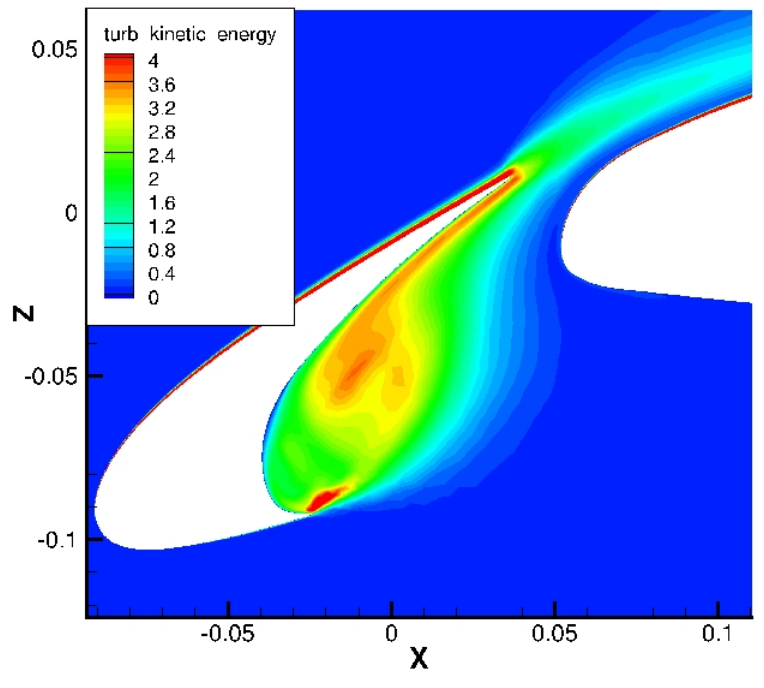

(a) Kato-Launder modification

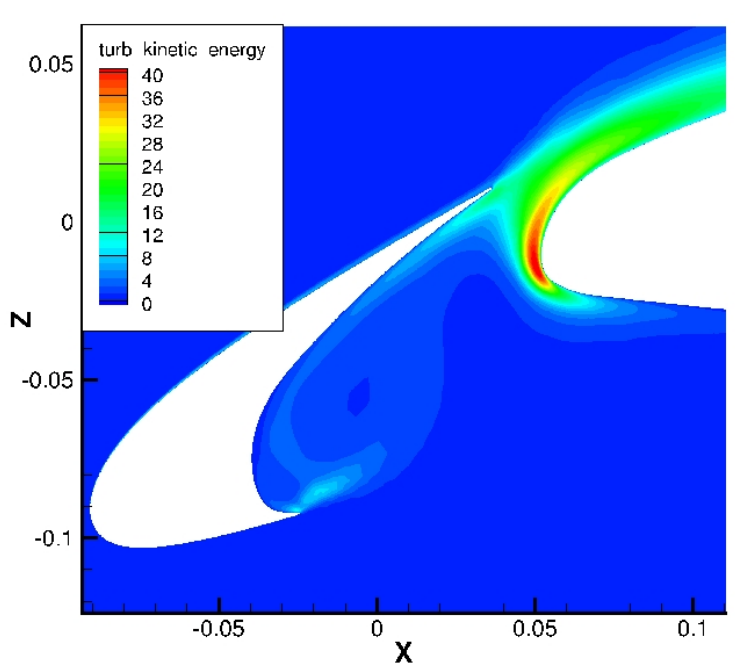

(b) Menter SST baseline

Figure 8. Turbulent kinetic energy in the slat-cove, $M=0.10$.

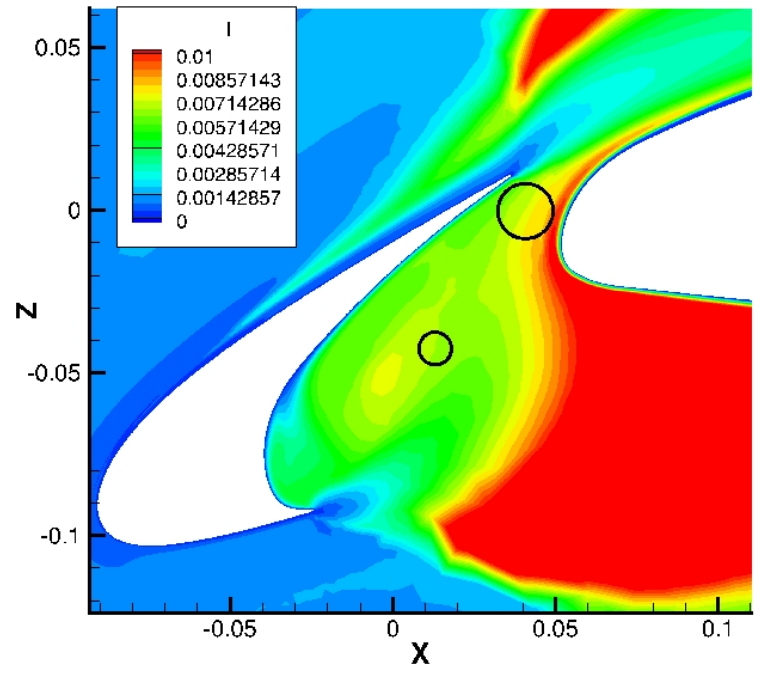

(a) Kato-Launder modification

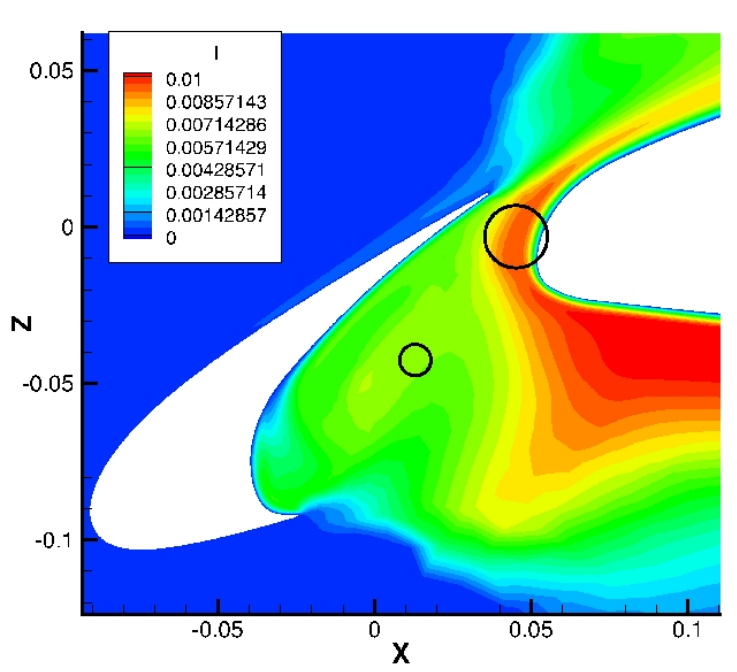

(b) Menter SST baseline

Figure 9. Length scale in the slat-cove, $c_{l} / C_{\mu}=6.0, M=0.10$; the circle indicate size of the length-scale (the radii are equal to the local length-scale at the circle origin). 


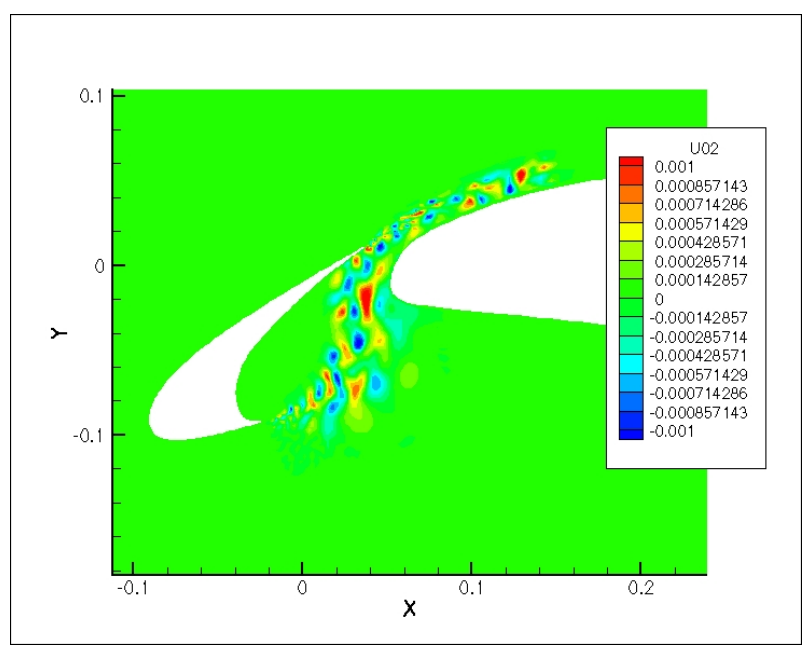

(a) Kato-Launder, $c_{l} / C_{\mu}=2.0$

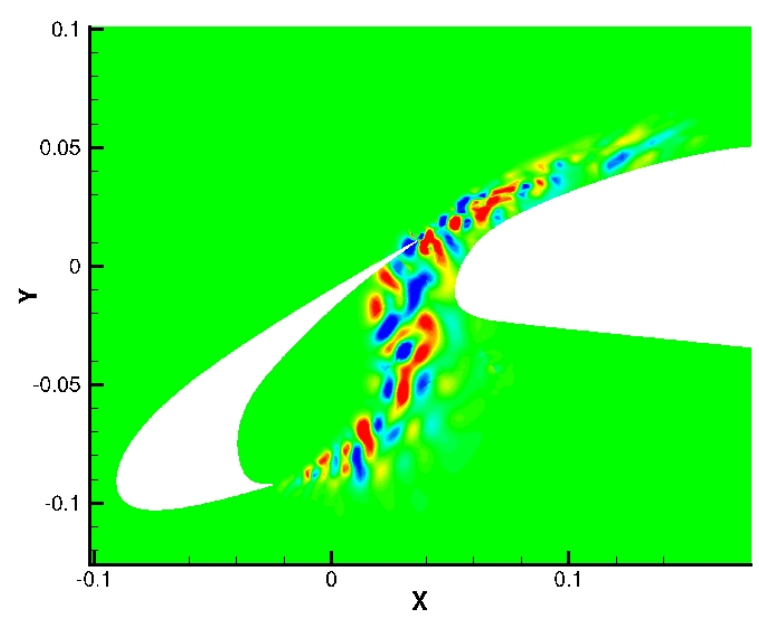

(b) Kato-Launder, $c_{l} / C_{\mu}=6.0$

Figure 10. Snapshot of the $v$-component of the generated stochastic field, Kato-Launder.

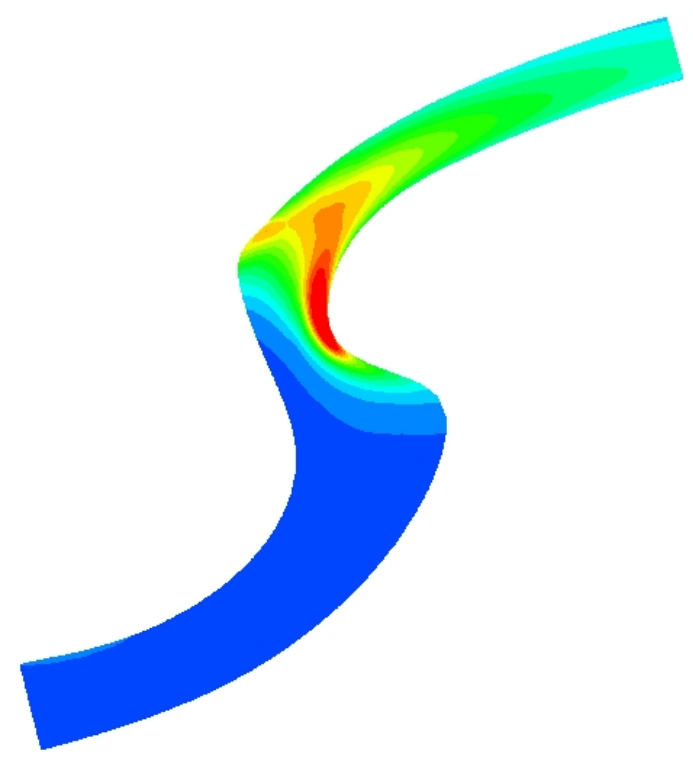

(a) Menter SST solution

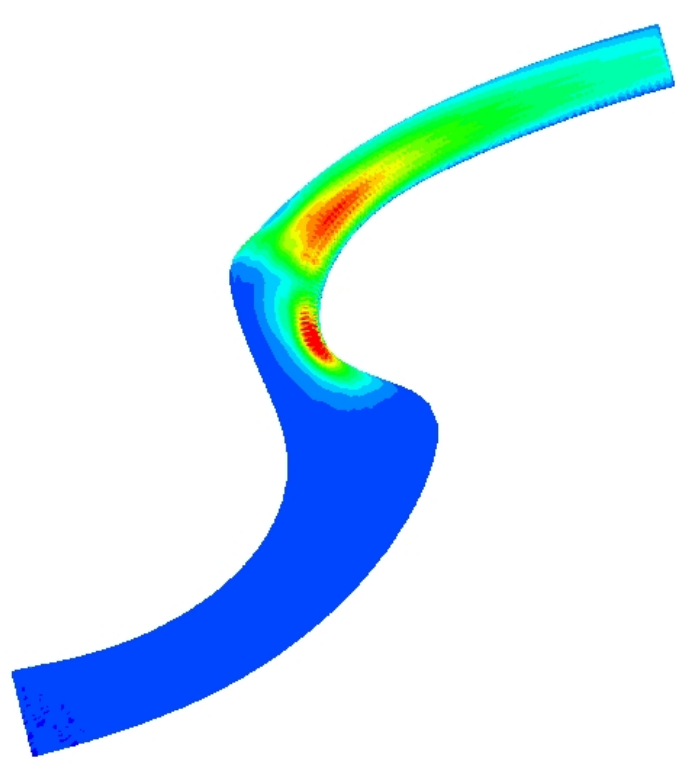

(b) Reconstruction from stochastic field

Figure 11. Turbulent kinetic energy in the slat-cove, $M=0.10$. 


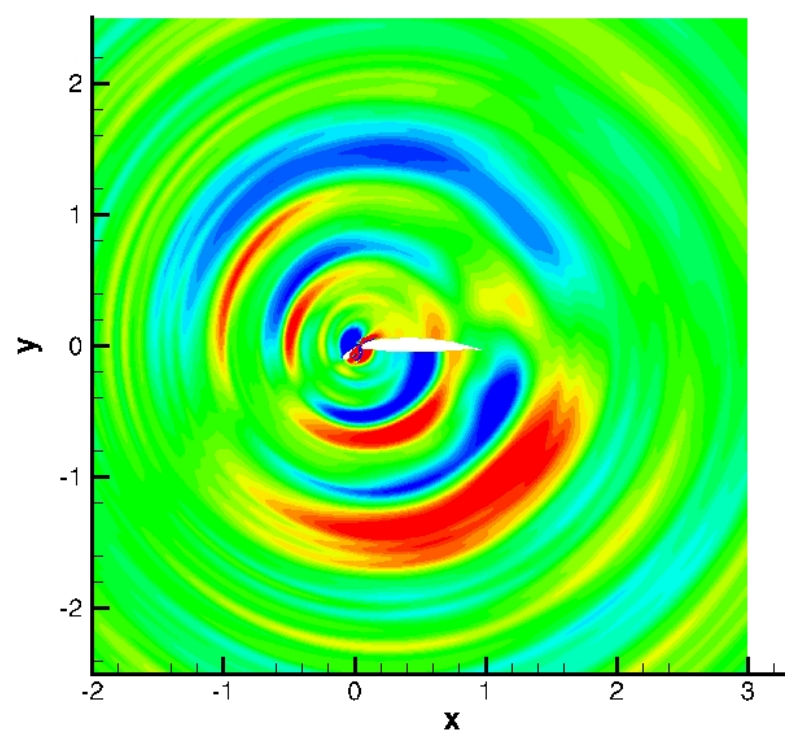

(a) With Kato-Launder modification

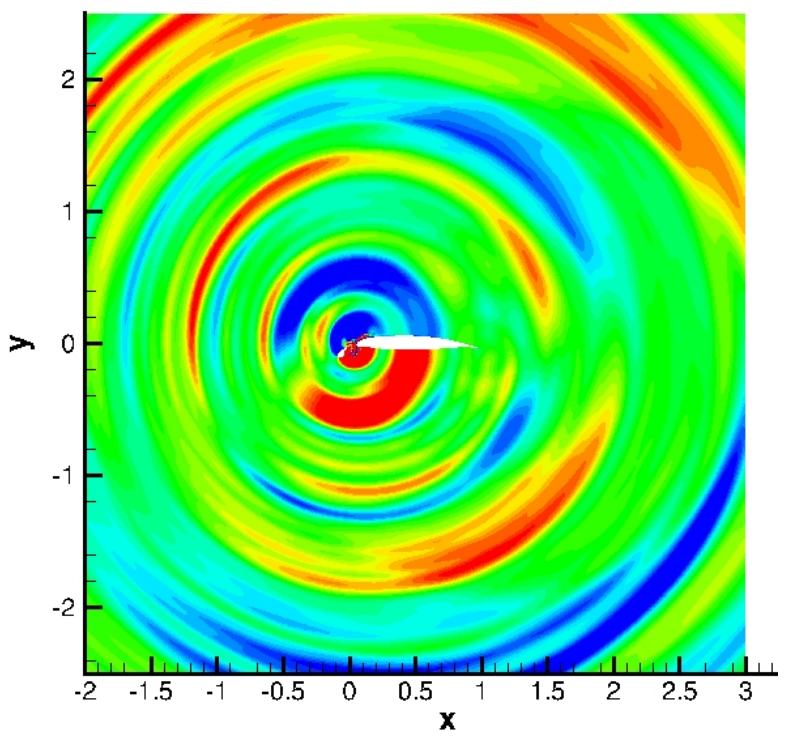

(b) Menter SST baseline

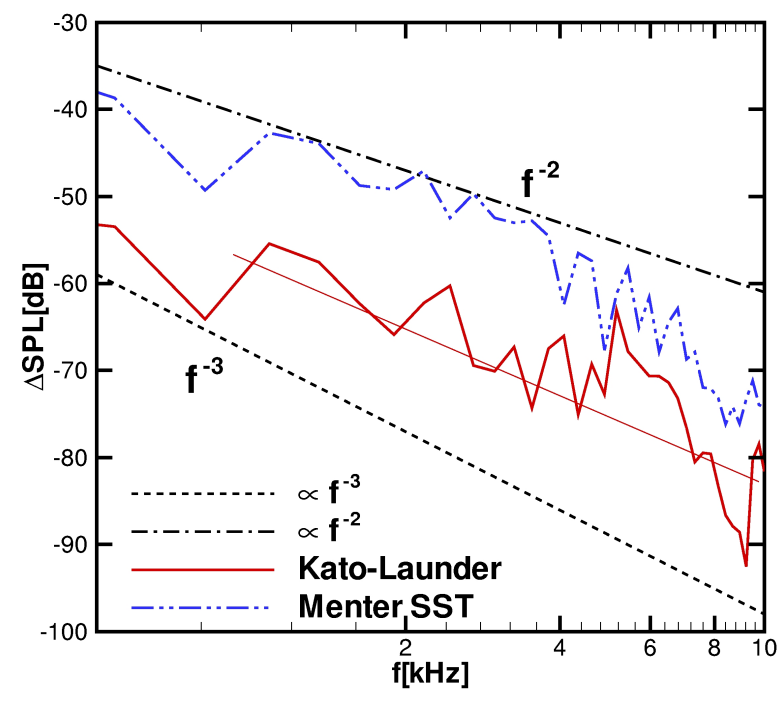

(c) Comparison of spectra

Figure 12. Sound pressure field and narrow band spectra, $M=0.10$. 


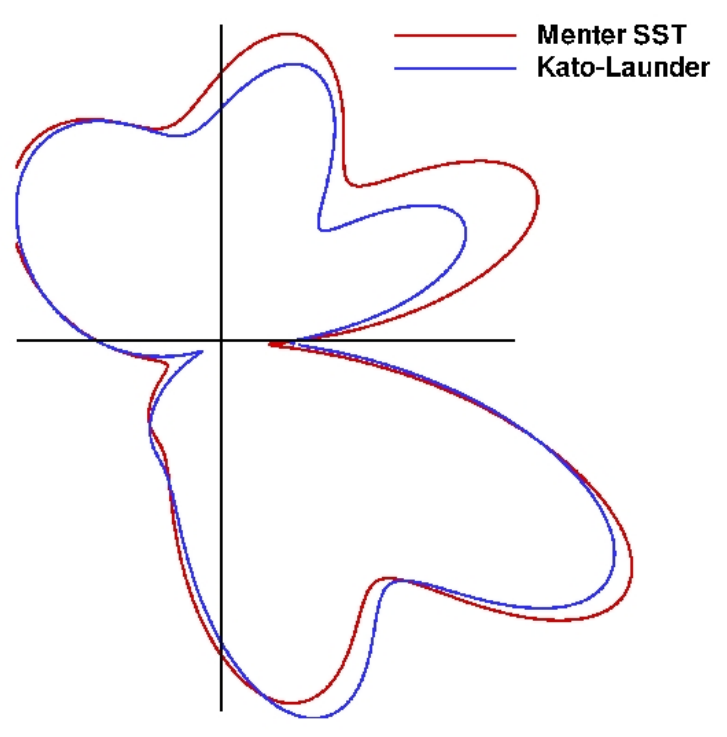

(a) $1 \mathrm{kHz}$

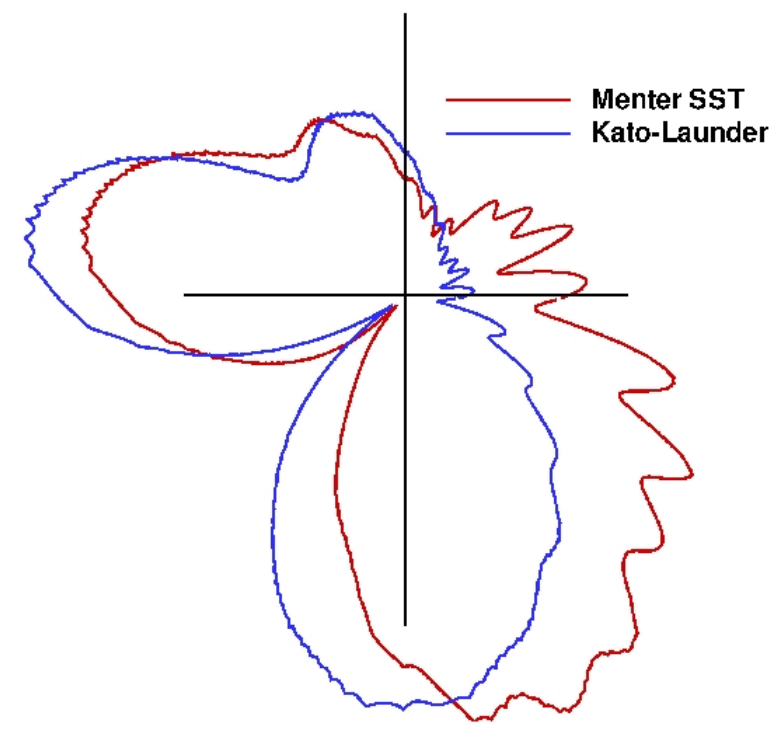

(c) $4 \mathrm{kHz}$

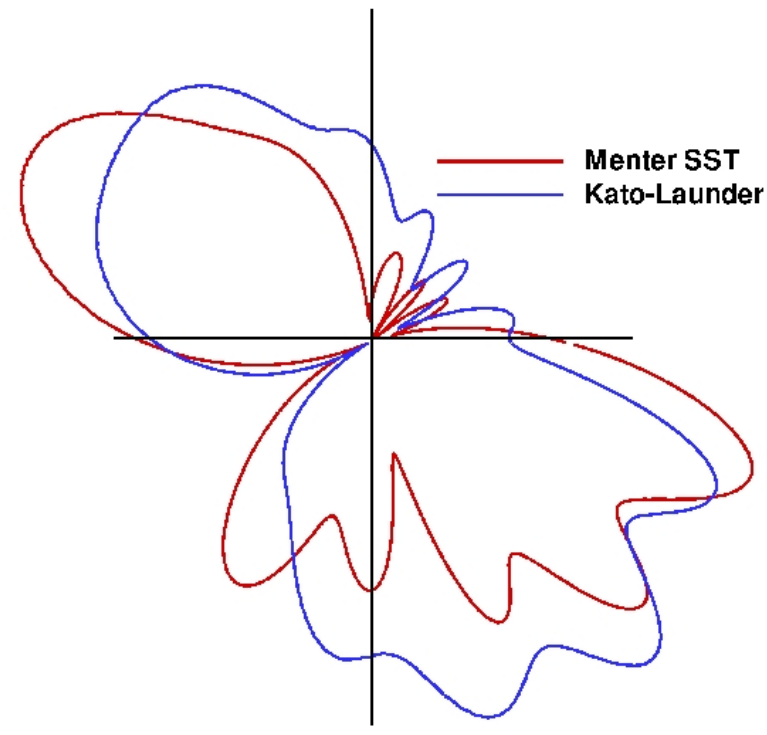

(b) $2 \mathrm{kHz}$

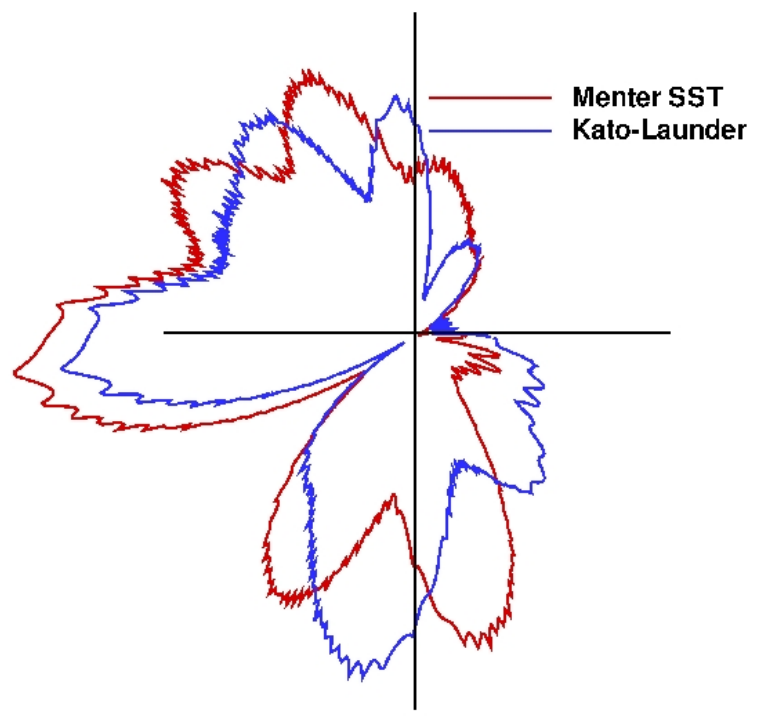

(d) $8 \mathrm{kHz}$

Figure 13. Directivity plots for the Menter SST model with/without Kato-Launder modification, the directivity circle is centered at the slat trailing edge and has radius $r=1.5 \mathrm{c}$. 
${ }^{22}$ Kato, M. and Launder, B., "The modelling of turbulent flow around stationary and vibrating square cylinders," 9th symposium on turbulent shear flows, Kyoto, Japan, August 16th-18th, 1993, 1993.

${ }^{23}$ Choudhari, M., Khorrami, M., Lockard, D., and Atkins, H., "Slat Cove Noise Modeling: A Posteriori Analysis of Unsteady RANS Simulations," 2003, AIAA Pap. 2002-2468. 